DOI 10.32370/IA_2020_01_6

\title{
Development of a Patenting and Licensing Strategy - principles of development of control and analytical algorithms taking into consideration new opportunities provided by quantum computers in light of analysis of readiness of a smart technology and its smart infrastructure for mass production and efficient integrated marketing
}

\author{
Yerzhan Kusparmakov \\ - Engineer, Inventor, Doctor of Philosophy (PhD) in Computer Science \\ - Professional Member of the New York Academy of Science \\ - Academician of the International Informatization Academy \\ - Advisor of the Russian Academy of Natural History \\ - Chief Product Manager at SAMSUNG Electronics
}

e-mail:kusparmakov@gmail.com

\begin{abstract}
Recently several new, in many respects - completely new areas of development of high-tech innovative products, which are unambiguously mass-market products, have emerged.

Such innovative areas may include the said and classified as - smart products and technologies, such products as - smart design, smart medical technologies, equipment and tool set, definitely including medical equipment and devices with efficient mobile applications, smart transport and smart transportation, smart manufacturing and smart manufacturing equipment, including special processing equipment with digital programmed control and the most perfect machining centers and flexible production modules.
\end{abstract}

Keywords: Patenting, Licensing, Quantum computers, Smart technology, Smart infrastructure, Mass production Recently several new, in many respects - completely new areas of development of high-tech innovative products, which are unambiguously mass-market products, have emerged.

Such innovative areas may include the said and classified as - smart products and technologies, such products as - smart design, smart medical technologies, equipment and tool set, definitely including medical equipment and devices with efficient mobile applications, smart transport and smart transportation, smart manufacturing and smart manufacturing equipment, including special processing equipment with digital programmed control and the most perfect machining centers and flexible production modules.

It also includes Smart workforce development.

Let us consider this whole process on the example of introduction of modifications of mobile secure coding of digital external storage media. 


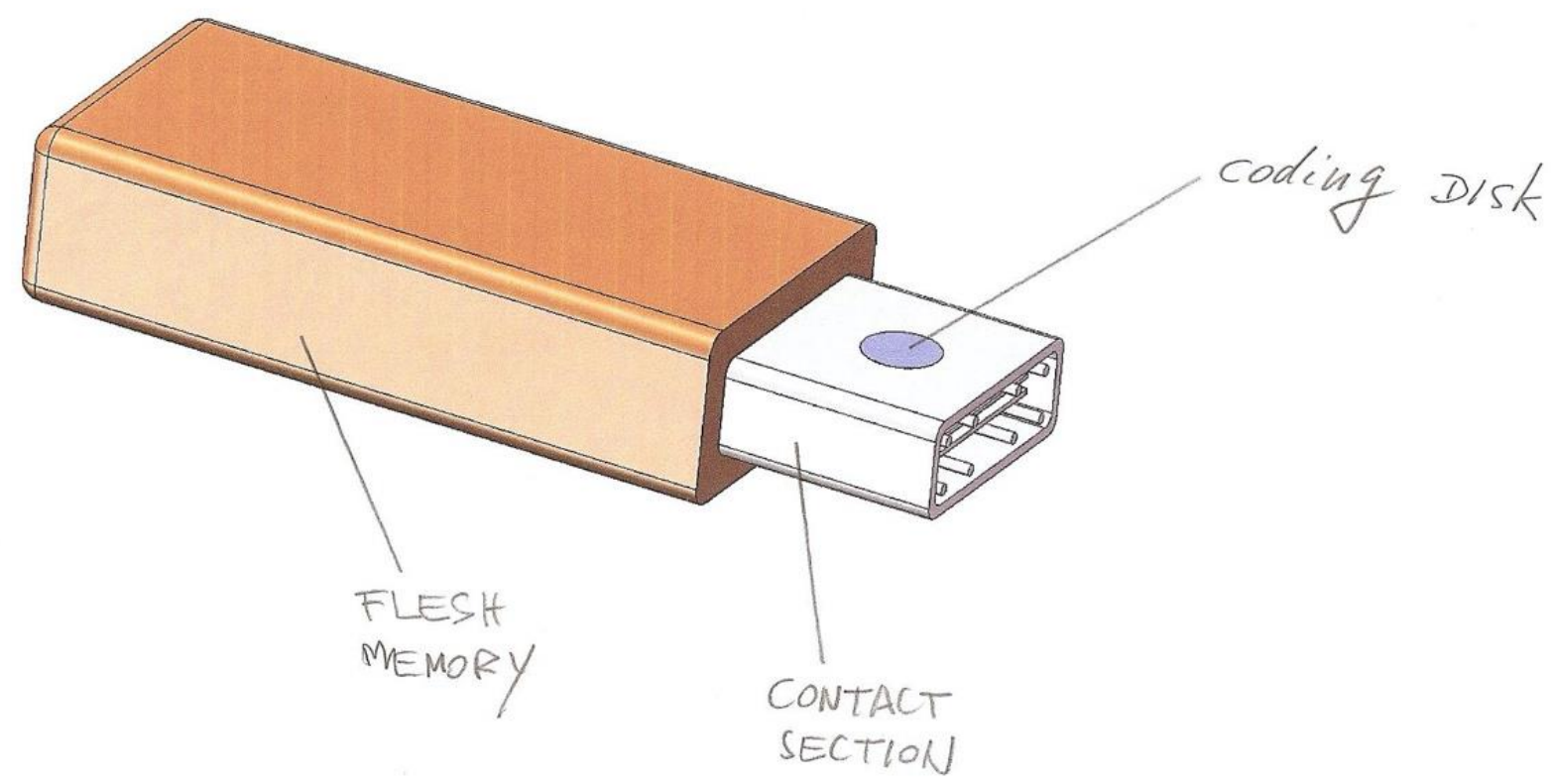

Figure 1, - an example of such an external storage medium modified by the innovative coding system is presented in the drawing

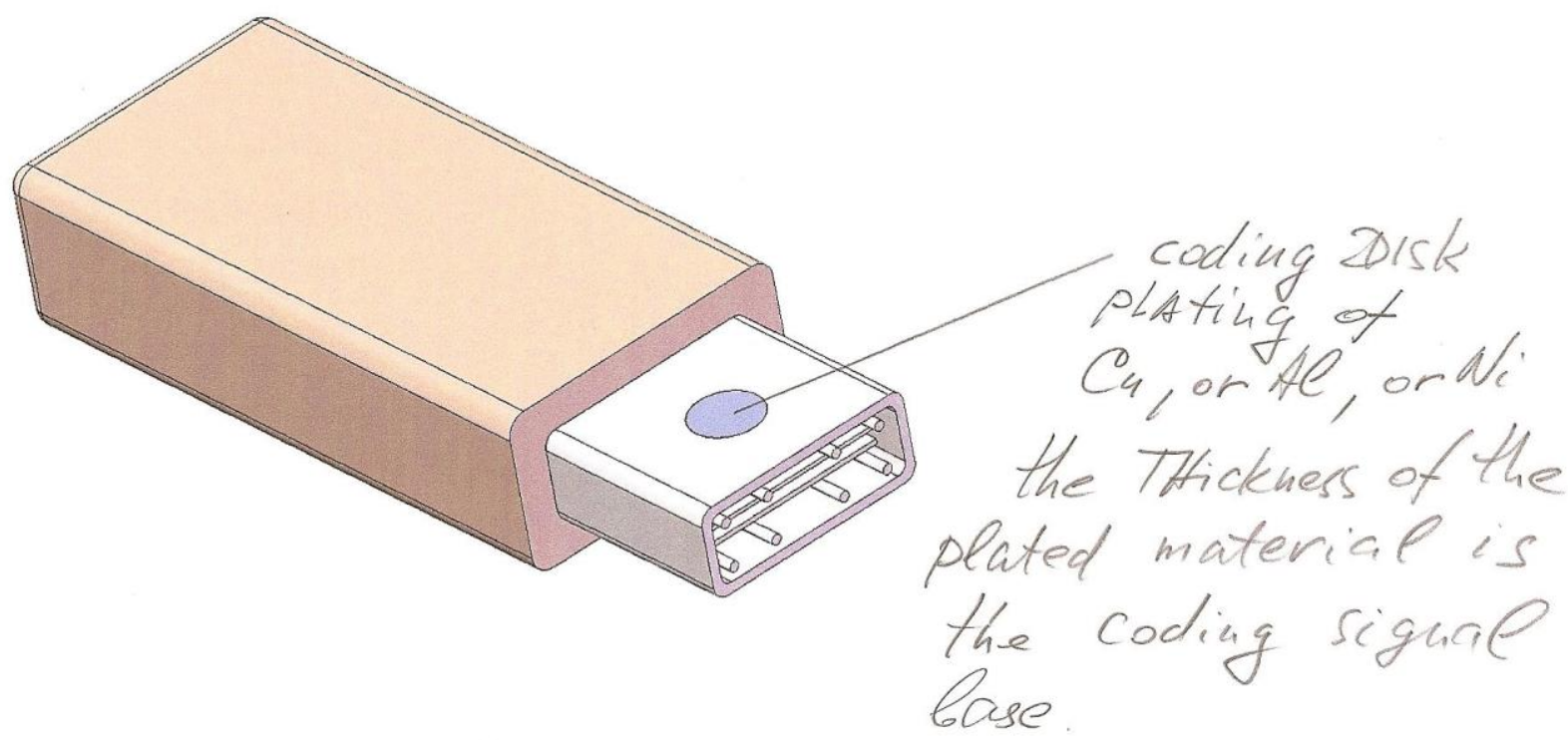

Figure 2, - an example of such an external storage medium modified by the innovative coding system is presented in the drawing as well

The principle of the coding system and materials used for its construction and their combinations are also shown in the drawing. 
A solution of such a coding and decoding unit, with all its simplicity, allows obtaining maximum accuracy of code identification, since the specified accuracy is determined by the accuracy of a thickness of a coding or decoding disk.

There are at least two methods of manufacturing and installation of this disk in a casing, - the first method is the method of manufacturing of a coding disk of precision foil, the second method is applying of a precision electrochemical coating in a groove made in the casing for the coding disk.

\section{General information}

All projects of this group of technical solutions are based on one method of coding and subsequent identification of record of a coding element.

The essence of the principle is in applying a coding coating or its technological equivalent on a secured object and further measuring the thickness of this coating, which determines coincidence or non-coincidence of measuring results with the code.

In case of coincidence of an obtained result with the set one there is positive identification of the coding element, in case of non-coincidence, - there is negative identification and stop or blocking of an operating cycle of equipment or information consumer, for example, - a computer. 


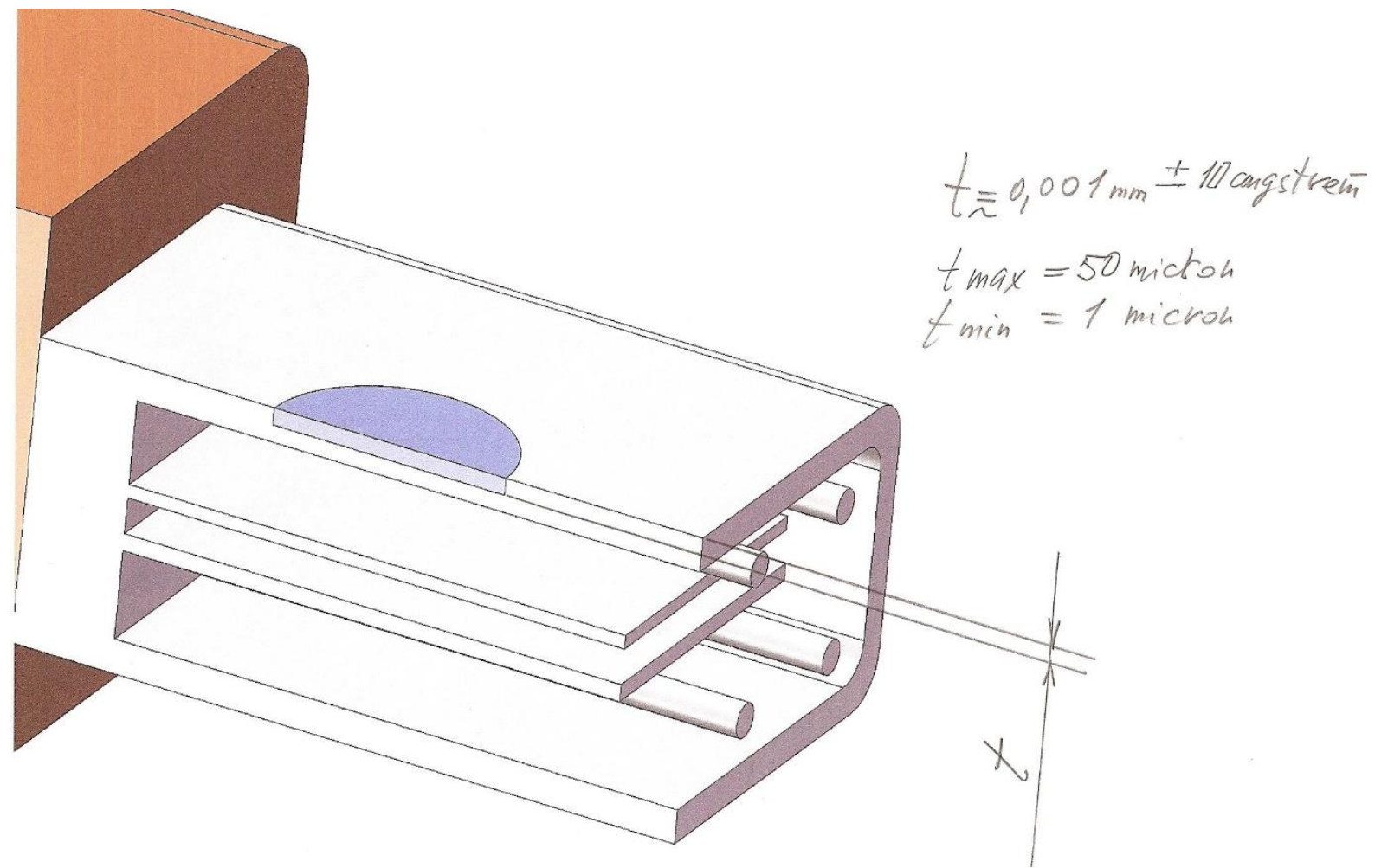

Figure 3, - an example of such an external storage medium with indication of technical details modified by the innovative coding system is also presented in the drawing

The most critical value determining the accuracy of coding or decoding is shown in the section, - the thickness of the disk - $t$.

The minimal thickness may be equal to 1 micron, the maximum thickness -50 microns.

\section{Additional technological characteristics}

Technologically matters of applying special coatings are solved, and this technology was checked many times on analogous tasks related to control of film thickness on solar arrays and in traditional semiconductor manufacturing.

Additional characteristics and possibilities of technology use, with regard to new conditions that emerged on the market of storage media and information tanks for the last year taking into account emerged new courses in technical solutions and technical systems of any levels eventually leading to synthesis of the so-called smart technologies and complex technical systems with elements of artificial intelligence and artificial neural networks. 
Patenting \& Licensing Strategy

$\underline{1}^{\text {st }}$ INITIAL STAGE OF PROJECT - NEW INTERNET TECHNOLOGY PRINCIPLES (RESEARCH)

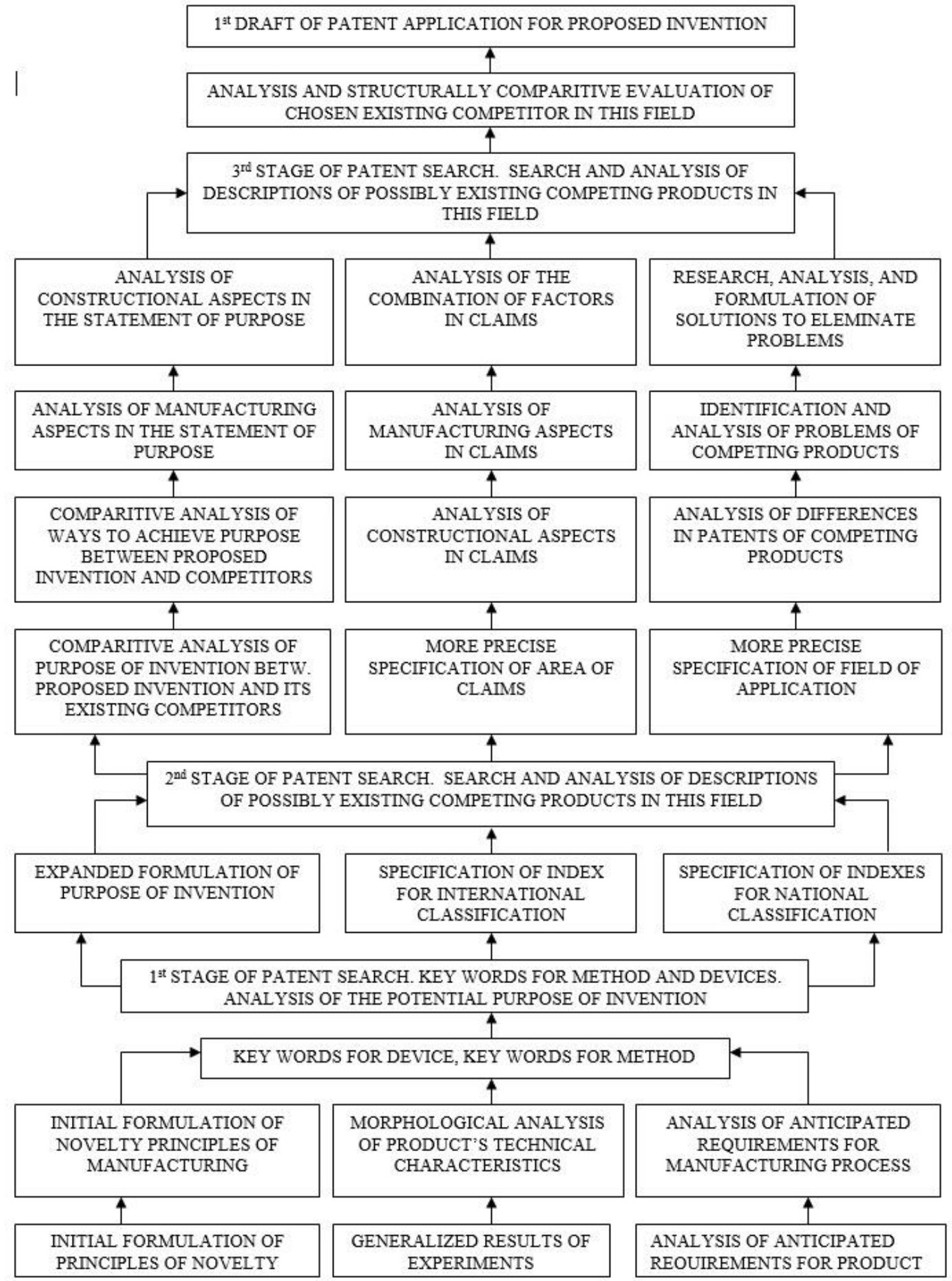

Figure 4, - an algorithm of using the patenting and licensing strategy at initial stages of project development is presented in the drawing 
Patenting \& Licensing Strategy

$2^{\text {nd }}$ INITIAL STAGE OF PROJECT - Codification Technology Principles and Internet integrated solutions DEALS WITH CORE TECHNOLOY THAT RELATES TO BASE TECHNOLOGY AND CONSTRUCTION PRINCIPLES OF PRODUCT ("BASE"), AS OPPOSED TO A SPECIFIC APPLICATION OF THE PRODUCT

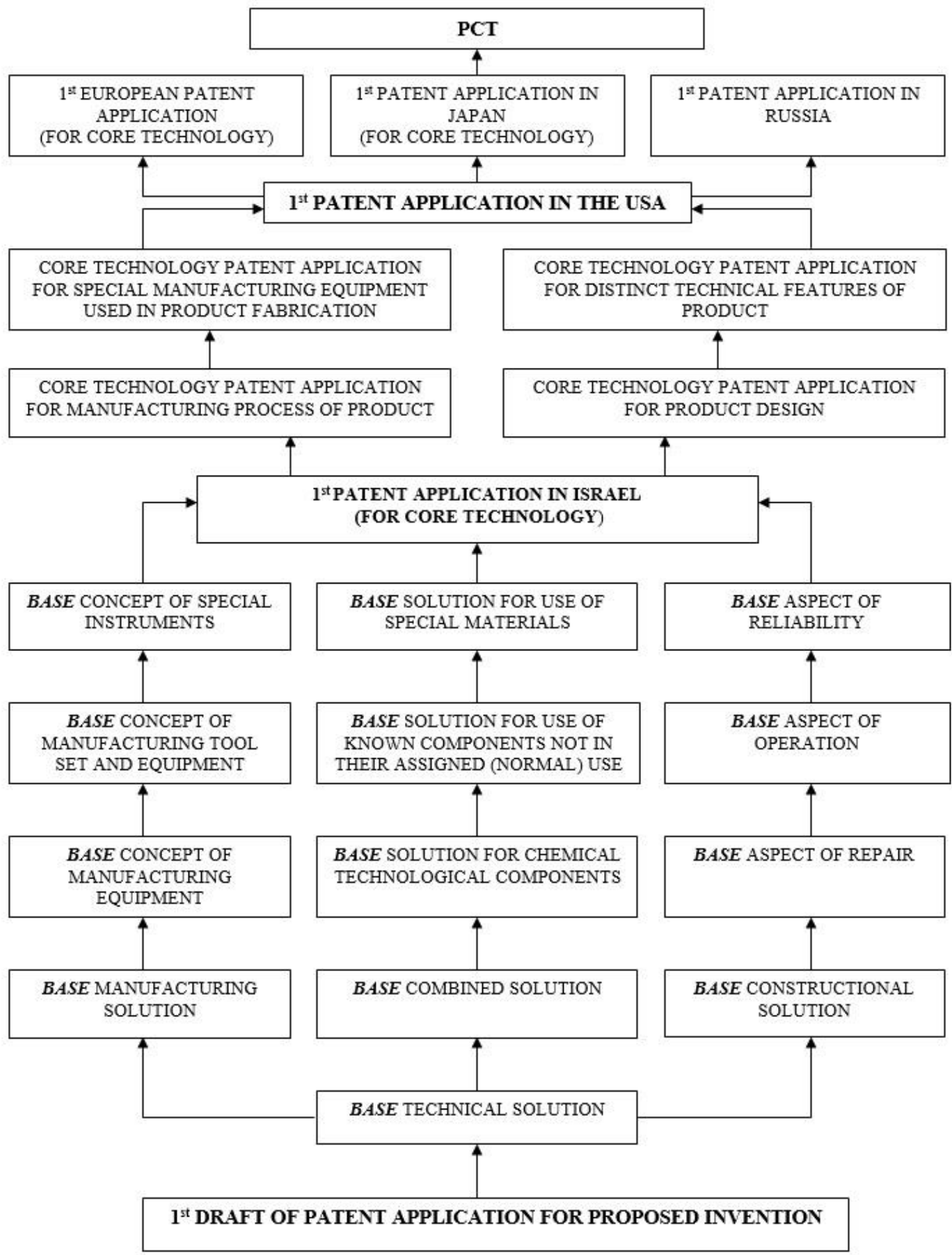

Figure 5, - an algorithm of using the patenting and licensing strategy at subsequent stages of project development is also presented in the drawing 
Patenting \& Licensing Strategy

Codification Technology Principles, Coder-Encoder system and Internet solutions and interfaces $3^{\text {rd }}$ INITIAL STAGE OF PROJECT (RELATING TO SPECIFIC APPLICATION)

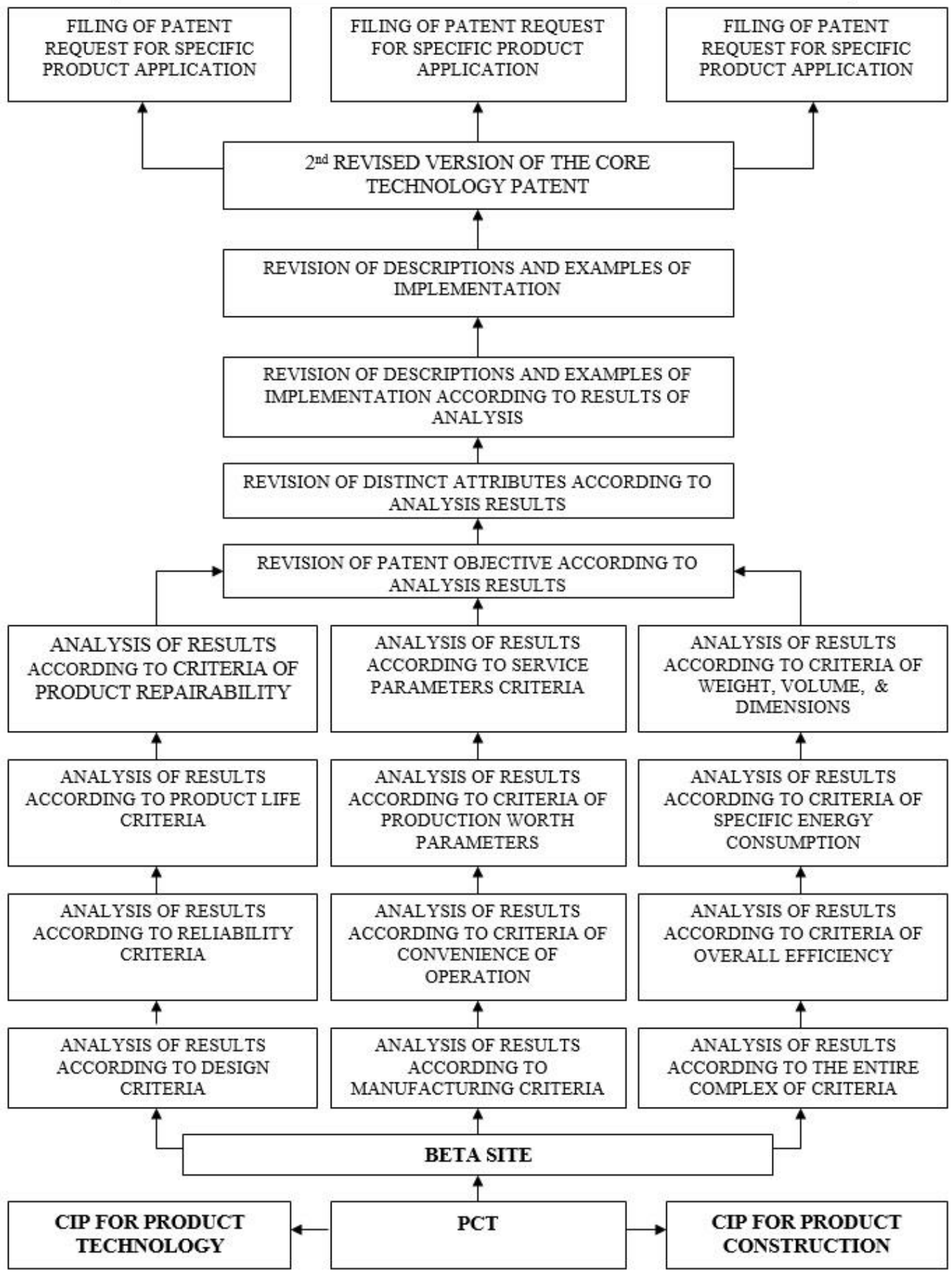

Figure 6, - an algorithm of using the patenting and licensing strategy at medium stages of project development is presented in the drawing 
Patenting \& Licensing Strategy

STAGES: DETAIL DESIGN FOR MASS PRODUCTION of CODIFICATION TECHNOLOGY PRINCIPLES and CODER-ENCODER systems FOR Internet solutions, - COMPONENTS PRELIMINARY TESTING DOCUMENTATION FOR MASS PRODUCTION

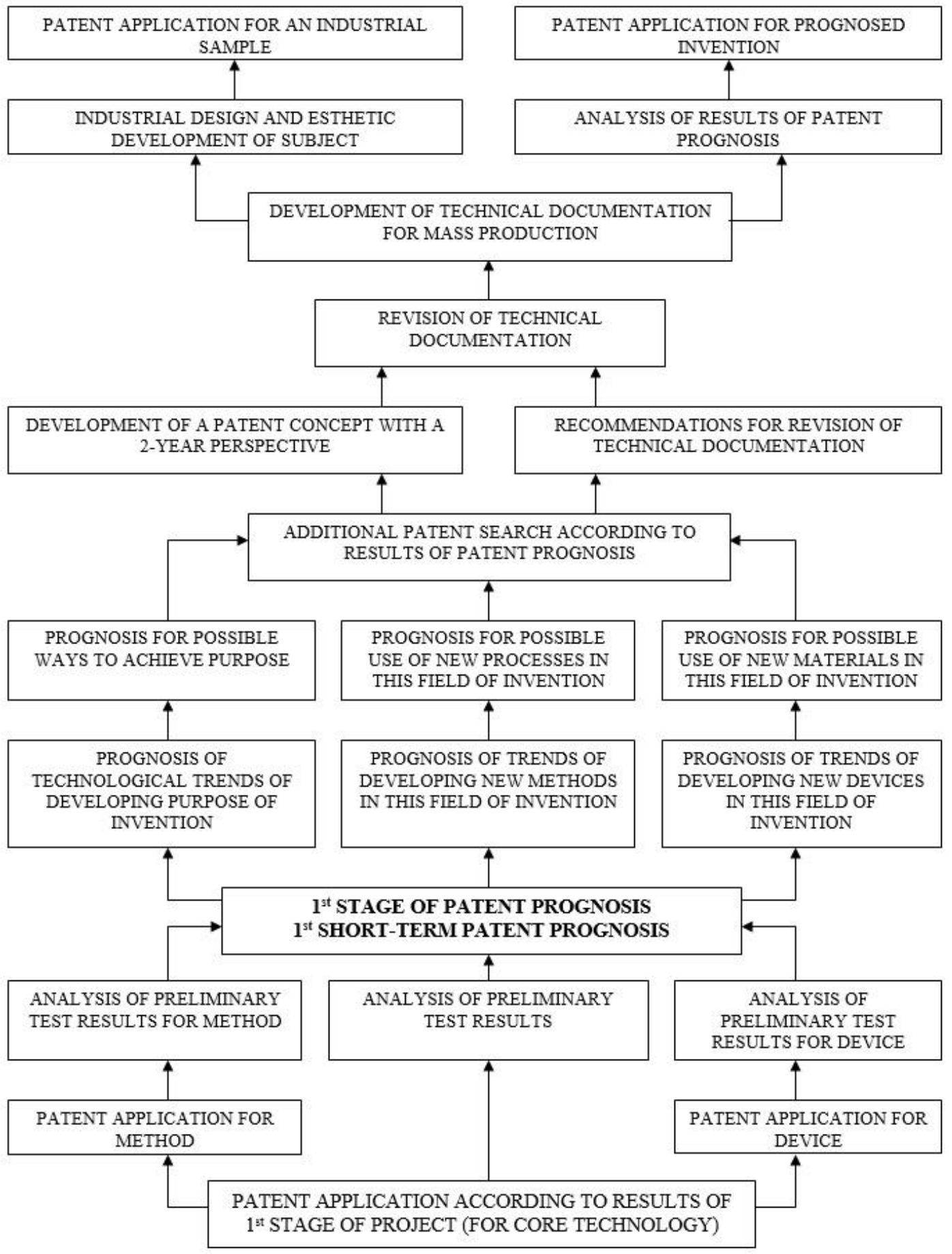

Figure 7, - an algorithm of using the patenting and licensing strategy at design stages for mass production is presented in the drawing 
Patenting \& Licensing Strategy

Codification Technology Principles, Coder-encoder system and Internet new solutions and interfaces STAGES:
TRIAL RUN OF MASS PRODUCTION
PRODUCT ACCEPTANCE TESTING
FULL SCALE MASS PRODUCTION

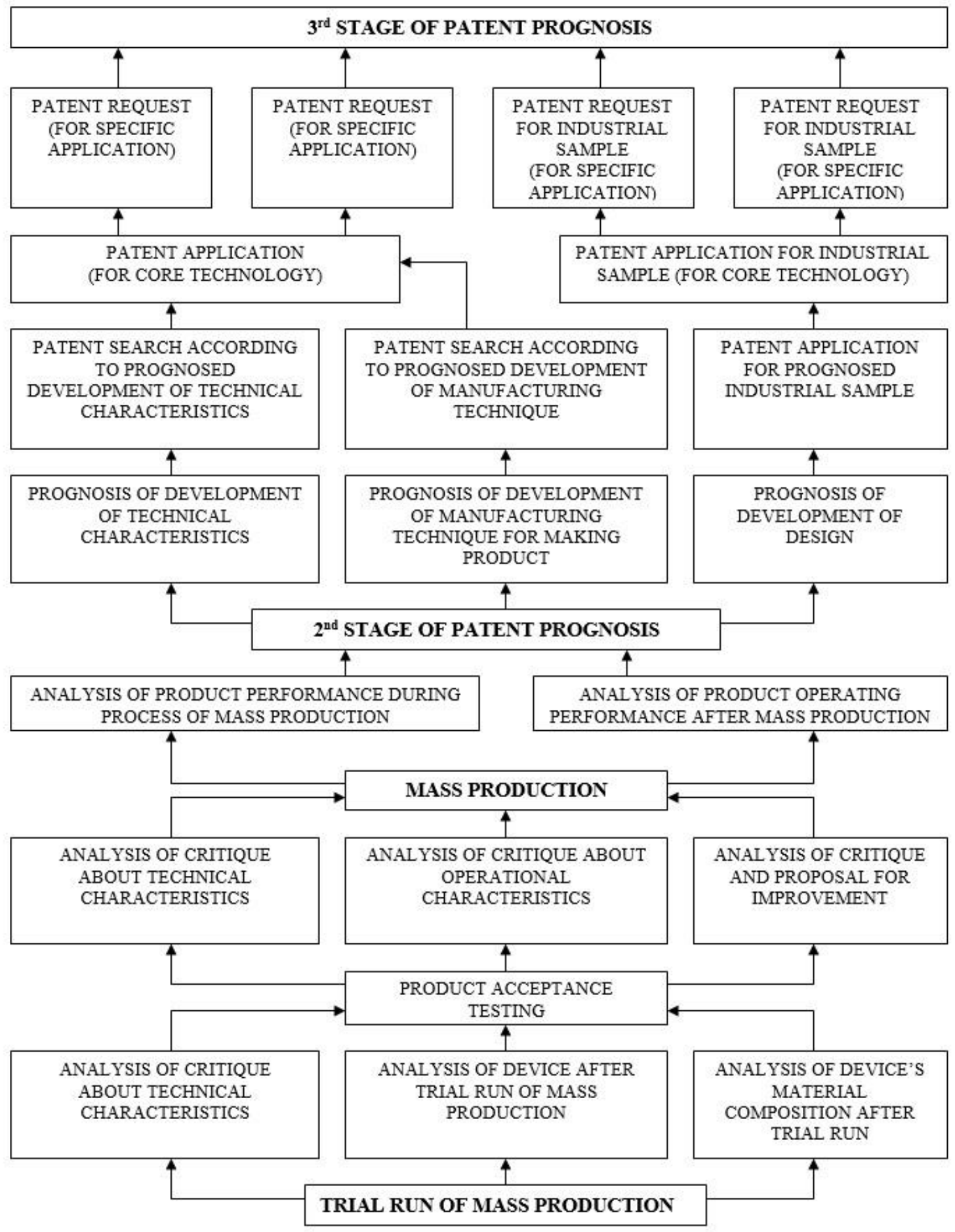

Figure 8, - an algorithm of using the patenting and licensing strategy at stages of mass production, stages of acceptance and qualification tests and after adjustment based on their results transition to full-scale mass production is presented in the drawing 
Patenting \& Licensing Strategy

CODIFICATION TECHNOLOGY PRINCIPLES in Internet solutions, programs and interfaces STAGES:

MASS PRODUCTION

ACTIVE MARKETING PHASE

PRODUCT IMPROVEMENT

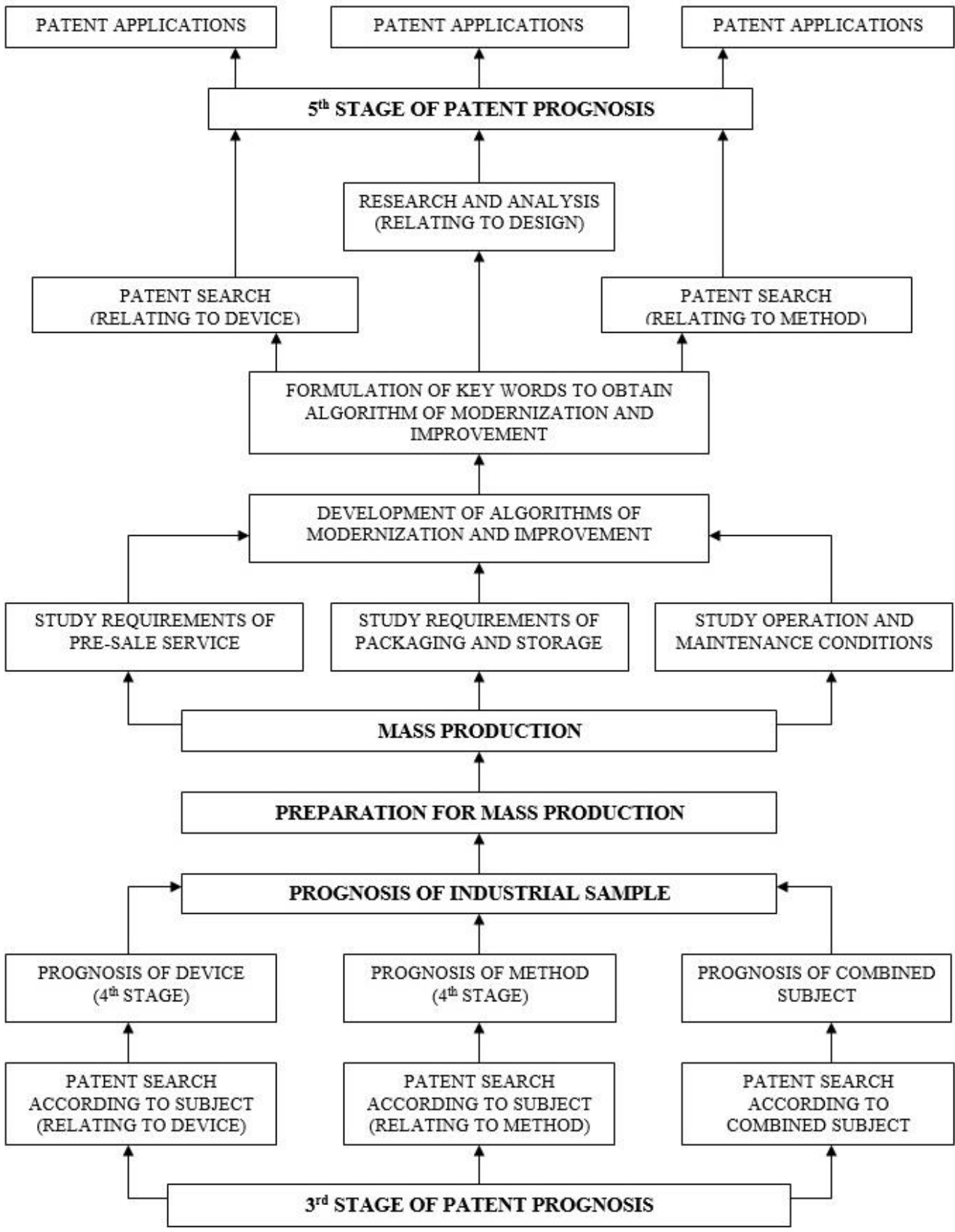

Figure 9, - an algorithm of development and introduction of the patenting and licensing strategy at the stages of project development is presented in the drawing, - such as, - mass production, an active phase of complete marketing and subsequent after them and parallel to them an integrative stage of optimization and modification of the product imparting the product properties and characteristics, - of a smart product manufactured by a smart 
technology, on smart equipment, using smart materials and components, using smart workforce within the framework of the smart production infrastructure

A special attention (in accordance with the algorithm) is paid to patent forecasting in terms of the device, in terms of - methods, in terms of - the combined and integrated object, to the extent practicable using elements of artificial intelligence with artificial neural networks in all component parts of the product infrastructure.

From the marketing experience after implementation of all aspects and features of mass production of a new product, analytical requirements are introduced to the algorithm to all elements of the marketing process service system, - study of pre-sale service, study of packaging and storage requirements, as well as study of requirements and conditions (especially technical requirements and technical conditions) to a procedure and methodology of repair and maintenance.

All these types of activity provide for an integrative combination of all above mentioned aspects and based on them possible objective and task setting on optimization and further project development.

\section{Comments to algorithms (shown on the drawings above)}

Figure 4, - an algorithm of using the patenting and licensing strategy at initial stages of project development is presented in the drawing.

Specific issues solved on this fragment of the algorithm:

- formulation of general novelty of the product;

$\circ$ formulation of general operating performance of the product;

○ formulation of a technical specification of the product;

- formulation of a manufacturing specification of the product;

○ formulation of basic technical requirements to the product and its production;

○ formulation of patent search requirements;

○ formulation of technical characteristics of the product and its potential of comparative novelty and potential for the next 5, 10, 15 years.

Figure 5, - an algorithm of using the patenting and licensing strategy at subsequent stages of project development is also presented in the drawing.

Beginning of the algorithm - development of the first option of patent application on the new product with due account for all characteristics and results of all types of patent search, as well as market researches.

Completion of the algorithm - development and filing an international patent application with due account for all results of market researches and its requirements to the new product. 
A nature of basic conceptual solutions on all characteristics and properties of the new product are constantly analyzed in perfection of the algorithm.

Figure 6, - an algorithm of using the patenting and licensing strategy at medium stages of project development is presented in the drawing.

Beginning of the algorithm - development and filing an international patent application with due account for all results of market researches and its requirements to the new product, as well as development of the new product project and its applications in the format - program, system and associated method on the method - CIP, - continuation in part.

System analysis of results of pilot production and all types of tests.

Completion of the algorithm - preparation and filing system applications for supposed inventions, on materials of pilot production, on results of tests and on results of system analysis of all obtained results of this stage of production and implementation of the new product project.

Figure 7, - an algorithm of using the patenting and licensing strategy at design stages for mass production is presented in the drawing.

Beginning of the algorithm - preparation and filing system applications for supposed inventions, on materials of pilot production, on results of tests and on results of system analysis of all obtained results of this stage of production and implementation of the new product project.

Completion of the algorithm - preparation of materials and filing an application for obtaining an industrial sample for the new product.

Apart from that, this algorithm included all phases and stages of development of technical documentation, starting from stages of terms of reference, technical proposal, draft design, technical project, detailed design, corrections of documentation based on results of tests and pilot production.

Also, this algorithm included stages of patent search on arrays of world information with due account for results of tests and their structural analysis.

Figure 8, - an algorithm of using the patenting and licensing strategy at stages of mass production, stages of acceptance and qualification tests and after adjustment based on their results transition to full-scale mass production is presented in the drawing.

Beginning of the algorithm - mass production of the new product

Completion of the algorithm - 3 stages of patent forecasting, for detecting courses of optimization of the new product. 


\section{Theory of Inventive Problem Solving (TIPS) and Algorithms of Inventive Problem Solving (AIPS)}

Theory of Inventive Problem Solving and all known its derivative Algorithms of Inventive Problem Solving were created in the USSR and in the period from 1945 to 1947, where special emphasis was not laid on commercialization of innovative solutions, one may even say that at a certain stage of creation of the Theory and Algorithm of Inventive Problem Solving the issues of commercialization were ignored deliberately in favor of purely technological options of innovative solutions, absolutely detached from the real economy and in majority cases from the real life.

As the result of such short-sighted and one-sided organizational model of development of the innovative process, which took place at that time, inventors, who grew up and were brought up on classical TIPS and AIPS approaches and principles turned out completely unprepared for peculiarities and methods of competitive fight under the conditions of the modern society with free competitive economy, especially with gradual transition to system smart technologies, especially using elements of artificial intelligence and artificial neural networks.

They are willing to and like to invent, but are not prepared for and are not able to earn on their inventions, in order to get worthy compensation for their talent and creative work.

It is especially important to evaluate the necessity of beginning of the innovative process and understand, and even better to estimate all possible options of sequence of events in the commercialization process.

For this purpose, we find it expedient to use a system of assessment algorithmic criteria and tables represented by analytical assessment sheets, the algorithm of patenting and licensing strategy consisting of 6 group elements of assessment of criteria and parameters of an emerged innovative idea or initiative and process of their development and implementation.

Due to emergence of new formats of recording and reading on optical storage media using blue lases, and due to beginning of production of multilayer optical disks, based on the same technology, the proposed principles and technical solutions on secure coding have acquired even more value, since the quantity of recorded information on each disk increases and absence of protection leads to more loses of secret and confidential data.

In addition to already transferred information it is necessary to specify possibilities on coding each layer in multilayer disks, in which each level of record layers is coded, which is a substantial improvement of the formatting system of the optical storage medium in threedimensional expression and is a tool of ensuring (for especially important and secret information) local selective coding of information within one disk. 


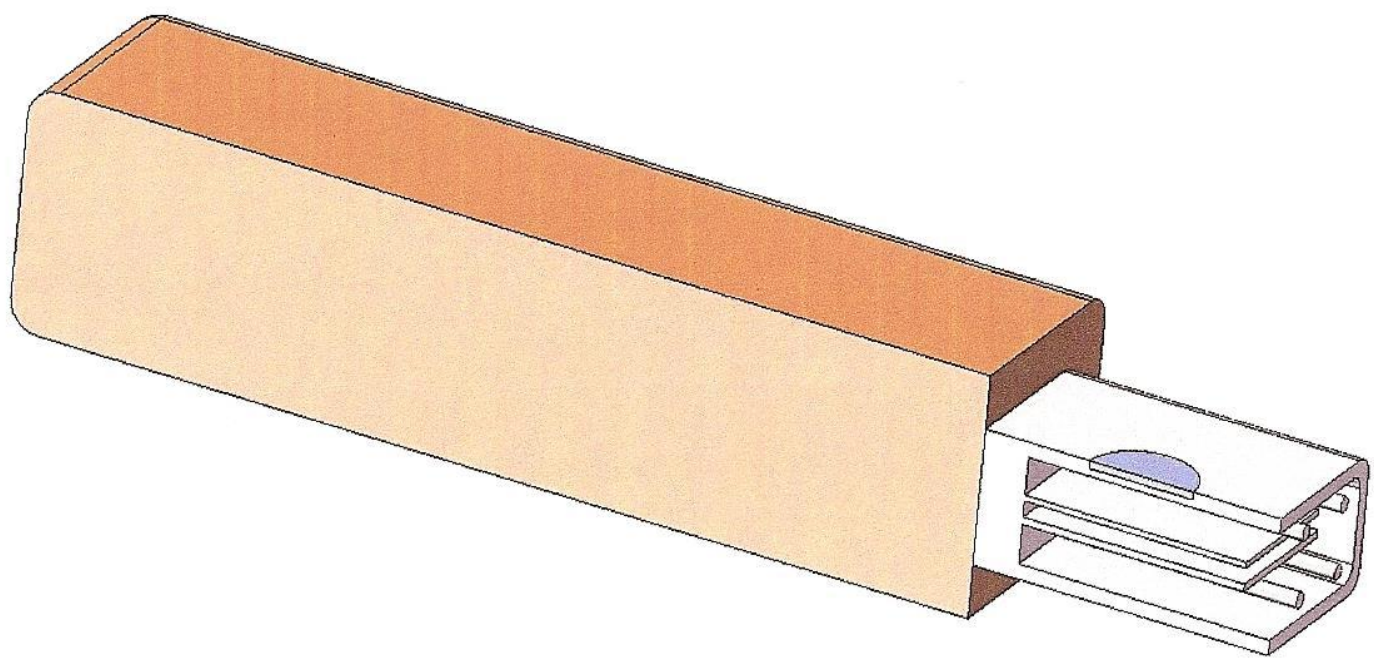

Figure 10, - a model of a storage medium in axial section is shown in the drawing

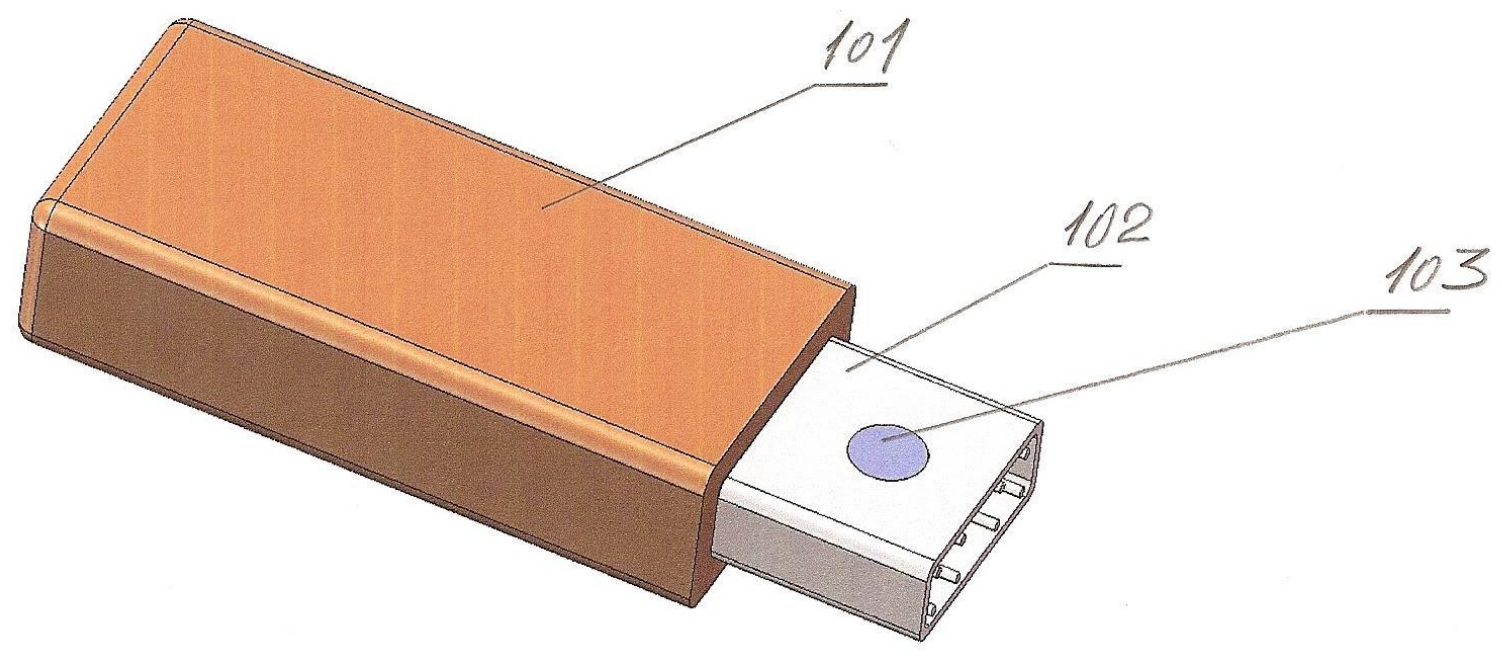

Figure 11, - a three-dimensional model of a storage medium is shown in the drawing 


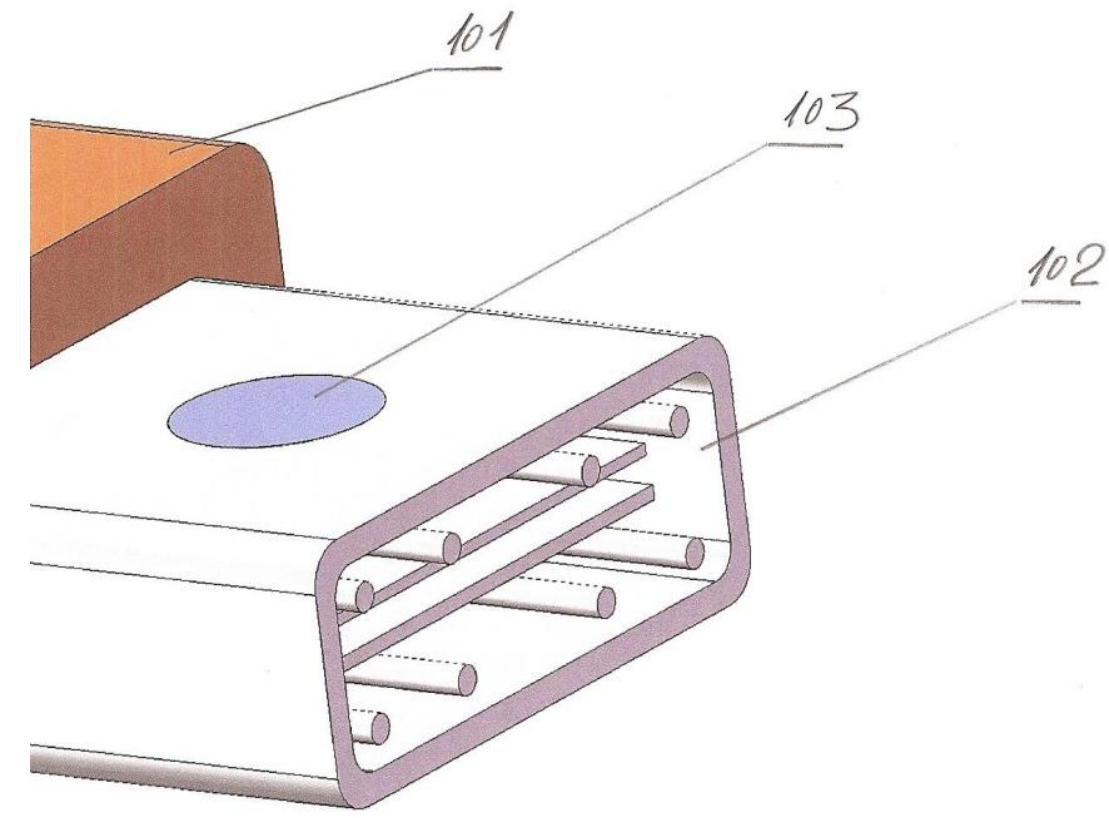

Figure 12, - a fragment of the three-dimensional model of a storage medium in the region of a flat plug connector with a coding and decoding disk is shown in the drawing

The following are figured in drawing 11 and 12:

- 101, - a casing of the storage medium;

- 102, - a flat plug connector;

○ 103 , - a coding disk.

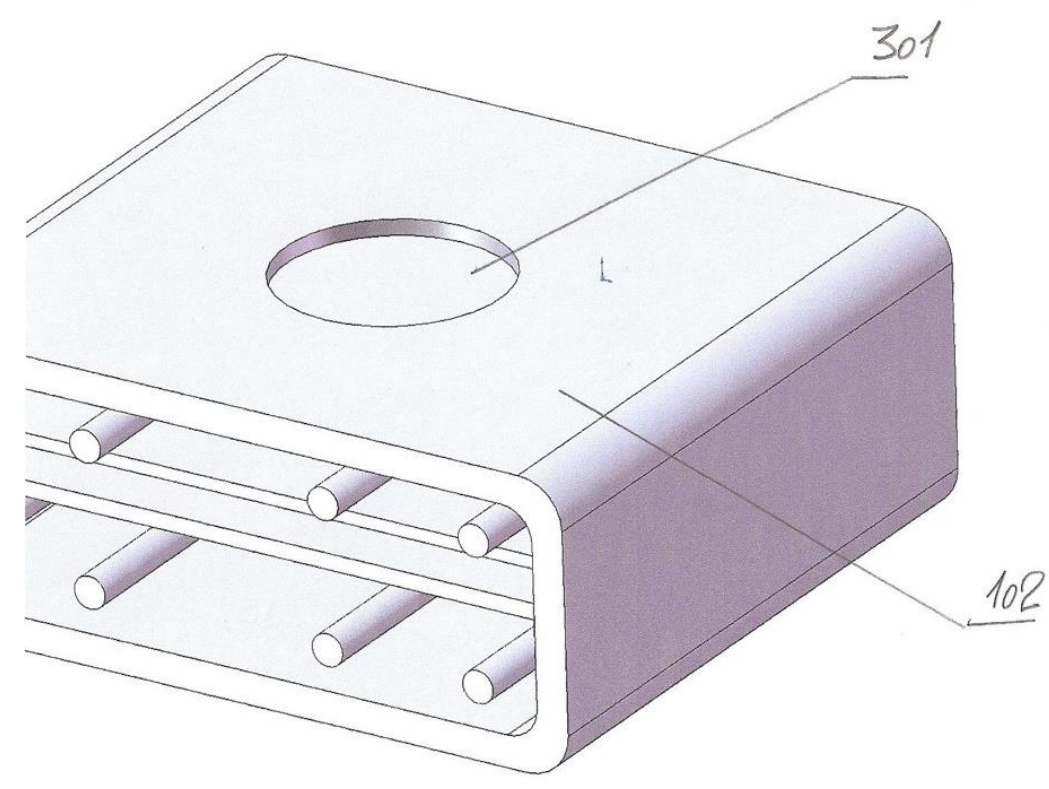


Drawing 13, - a fragment of the three-dimensional model of a storage medium in the region of a flat plug connector with a coding and decoding disk is shown in the drawing prior to installation of the coding and decoding disk

The following are figured in the drawing:

- 102 , - a casing of the flat plug connector;

- 301 , - a bore in the casing for installation of the coding disk in any design option.

As it is seen from all presented models of the storage medium in figures 10,11, 12 and 13, in terms of design the coding and decoding system is quite simple, which enables its integration even on storage media made without such device and such functions.

This implies an additional opportunity of using the newest technologies for increasing the marketing potential of the new product. The following technology can be cited as an example:

The method of production of a module for installation, cooling, management and control of energetically saturated optical and electronic systems including:

- treatment of a surface of a steel tape (roll) (metal can be specified instead of steel);

- application of photoresist;

○ development of photoresist;

- a rapid stream-like electrochemical coating with nickel in a directed flow of an electrolyte (2-3 microns thick);

- a rapid stream-like electrochemical coating with copper in a directed flow of an electrolyte (25-35 microns thick).

"Since this technological phenomenon is the main basic distinction and makes a package of substantial advantages of the proposed innovative method", - the author considers it necessary to give some explanation to this phenomenon.

The rapid stream-like electrochemical coating is a developed galvanic process in a selectively oriented directed flow of an electrolyte with a constant, electrolyte updating electrolyte recirculation system, which (the recirculation system) includes:

- a capacity with an electrolyte with certain parameters of a maintenance mode of the electrolyte in the capacity, such as:

○ concentrations of nickel and copper in the electrolyte

$\circ$ temperatures of the electrolyte

- acidity or alkalinity level of the electrolyte density of the electrolyte

- electric resistance of the electrolyte (conductivity)

0 owing to the abovementioned advantages of the technology and used materials there is no need in using organic additives to the electrolyte - clarifying agents;

○ a pump with a filter; 
- anode for stream-like metallization, which has soluble in this type of the electrolyte and insoluble in this type of the electrolyte components.

Components installed consecutively in the direction of the motion of the electrolyte, notably that the insoluble component was made of a composite, graphite and current-conducting tissue (like ETHANE). The insoluble component is located in parallel to a metallized area and last in the direction of the motion of the electrolyte and first before a covered surface (cathode). Both components are connected to a positive electrical potential as well as both components have a selective regulated hydraulic permeability for the electrolyte.

In the anode there is also a system of even distribution of the electrolyte on the plane of the soluble component, which is automatically repeated on the insoluble component and consequently on the metalized area - cathode:

○ photoresist removal;

○ iron etching, from one side, for the half of the thickness of the steel tape

- removal of etching products from the surface by aerodynamic and thereupon hydrodynamic effect;

○ pressurization by a free-flowing polymer compound, - in a such technologic procedure:

○ filling with a monomer

- subsequent layer-by-layer polymerization

○ thermal stabilization;

0 iron etching from the second side of the tape (with the same distinctions);

○ pressurization from the second side (with the same distinctions);

- application of a protector on electrically conductive structures;

- a coating in a vacuum of all heat-conducting structures - with a layered system of semiconductive nano-structured polycrystalline diamond films.

Such technological addition to traditional techniques and methods enables to expand spheres of introducing methods of marketing of the new product substantially.

\section{Organization of corporate security systems}

The proposed technology during organization of the security system of information flows within one corporation ensures security at several system levels, including both tracing in the real time system of a state and location of each disk, which is in the corporation.

When using the proposed methods of coding, for security of information on mobile external storage media, it is supposed to obtain the same advantages, as when using on optical storage media and information tanks. 


\section{Changes in the structure and boundaries of using the product created as the result of project implementation}

Thus, based on analogous solutions it is possible to create at least two projects with a large number of applications in each, - a technology project for coding optical information tanks represented by a disk, including a relevant analytically-sensing device, which may in its turn have a variety of applications in different spheres and branches.

And the project for coding and security of information on mobile external storage media, including a relevant mobile or stationary sensor measuring-analytically-comparative device, also having a variety of applications and design-models.

\section{Additional devices and systems, which can be created on the basis of the same fundamental technologic solutions}

Upon demand of a consumer of projects the project package may include a section, relating to additional or special devices, using which the whole corporate system of safeguard and security of information flows is formed within one corporation or a group of corporations or (according to Russian specificity, - state corporations) individual scientific research establishments, research institutes and large institutions in the healthcare system.

An information security system can be created as a special product not only in the sphere of storage, but in the operative sphere, when transferring commands and signals in the setting of army units and commands, and especially, in the setting of the - sea fleet.

In modern conditions, when information is concentrated in relatively very small sizes and capacities of devices for its storage, possible harm from unauthorized or criminal entry to these arrays of information can be prevented or localized using creation of a special infrastructure of the specified security systems, which can be standardized within the specificity of this ministry, main directorate or structural corporate units and enterprises of the lower organizational level.

A series of drawings, - from drawing 14 to drawing 20 shows, as an example, applications of coding and decoding technologies in identification of a one-use tool in laser medical devices. 


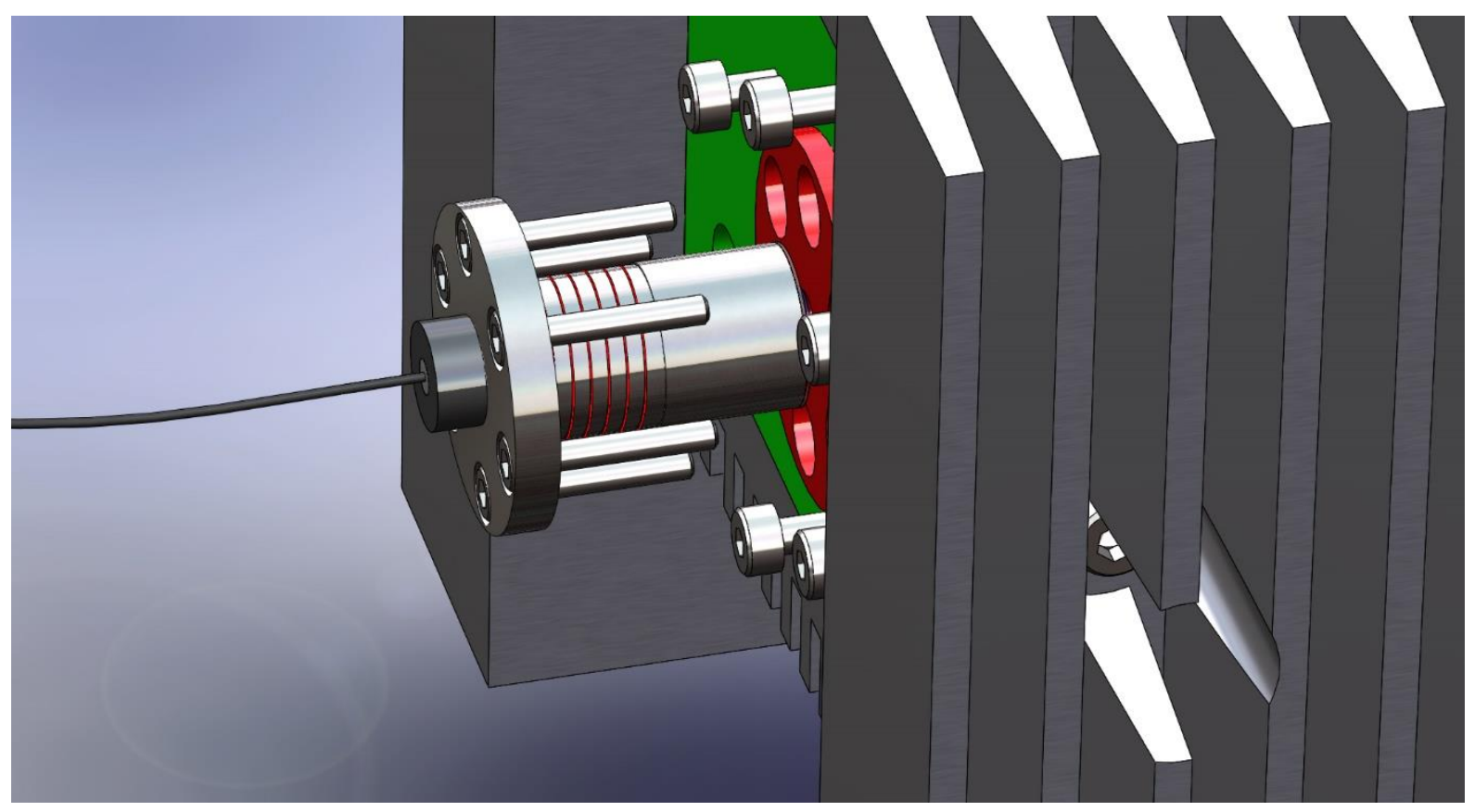

Figure 14, - a three-dimensional model of a docking device of a laser endoscope for installation and identification of one-use tool is shown in the drawing

Such combination of functions increases efficiency of operation of the system and substantially simplifies a design of the unit, which in its turn decreases its value and costs for operation and repair.

In its turn decrease in expenses while improving the quality gives additional warranties for more confident marketing for the new product.

\section{Confidentiality of information}

More in detail (in volumes going beyond this publication and illustrative materials to it) all necessary information can be provided during documentary and legal formulation of intentions of a potential consumer or a partner, after signing confidentiality agreements with him (by a mutually agreed, acceptable for both parties legal form).

A magnetic resonance method was applied for more complete presentation of existing physical bases of performance of coding and decoding operations of optical disks, a brief description of which is given below. 


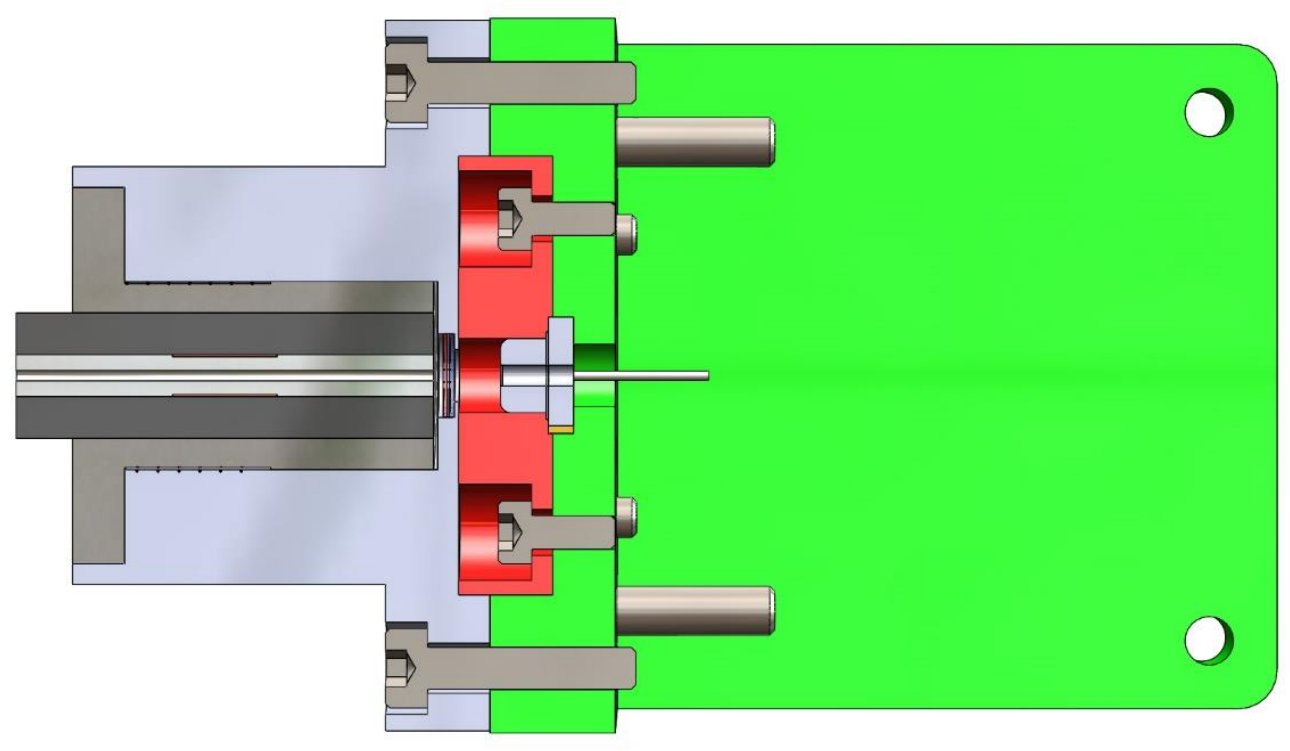

Figure 15, - a three-dimensional model of a docking device of a laser endoscope for installation and identification of one-use tool is also shown in the drawing. The model is shown in the axial section, where the system of functional interaction between a laser diode and optical cable, as the axis of one-use tool is seen

The system is maximally easy for reproduction and operation

\section{Brief description of the resonance method}

The method provides for creation of an alternating electromagnetic field in space, in which a studied sample is located. This field is an intermediate between a resonance circuit and the test sample.

On the one hand, the resonance circuit is an emitter (radiator) of this field, and on the other hand - an acceptor (a sensing element), of those changes in the electromagnetic field, which the tests sample makes.

Even in the absence of the test sample, the alternating electromagnetic field created by a solenoid is the sum of two electromagnetic fields, which change in antiphase to each other.

One field is generated by change of magnetic induction of the solenoid and have an electric curl field as a consequence (Maxwell-Faraday equation). The other one is generated by change of the electric field created by difference of potentials between extreme the most remote from each other solenoid coils (if the sample is placed inside of the solenoid) or difference of potentials between the closest to the surface coil of the measured sample and the sample itself 
(if the sample is located against the solenoid face), and has a whirling magnetic field as a consequence (Ampère's circuital law with Maxwell's correction).

Affected by the external alternating electromagnetic field in the test sample, depending on its nature, such electric phenomena as linear and whirling currents of conductivity, linear and whirling currents of displacement, as well as linear and whirling ionic currents (ordered motion of ions) may be induced.

In accordance with the superposition principle of fields these electric phenomena introduce distortions to the external alternating electromagnetic field.

These distortions are perceived by the solenoid of a resonance sensor. A resonance circuit that includes this solenoid, changes its behavior similar to that, as if it included additional elements: a condenser, inductivity and a resistor.

The aggregate of additional capacity, inductive and active resistances represents an additional impedance contributed by the test sample to the system, this attribute is measured by the resonance sensor.

Changes of parameters of the resonance circuit are reflected in the change of its amplitudefrequency response, namely, resonance frequency and amplitude of the circuit change.

Studying these changes, one may estimate impedance of the tests sample.

\section{Principle of processing of data obtained from resonance sensors}

The resonance sensor allows determining a value of a resultant impedance of the studied sample at an operational frequency of this sensor (see "Brief description of the resonance method").

This value by its self is low informative.

But everything ultimately changes, if we have a set of sensors with different operating frequencies.

In this case there is an opportunity of using a unique natural phenomenon observed in all types of substances: non-organic, organic and biological. This phenomenon resides in the fact that a substance changes its specific impedance depending on a frequency of an electric field affecting it, and this change depends upon a composition of a studied substance.

A rapidly developing in the recent time scientific field called Magnetic resonance spectroscopy - impedance spectroscopy studies this phenomenon and actively uses it.

In English-language sources it is more often called Electrochemical Impedance Spectroscopy (EIS) (impedance spectroscopy). 
Magnetic resonance spectroscopy - impedance spectroscopy is a method of researching various objects, based on measurement and analysis of dependences of impedance upon frequency of the alternating current.

Different objects and processes are characterized by different dependences of active and reactive impedance on frequency, which makes possible solving an inverse problem obtaining information about these objects and processes through analysis of frequency characteristics of their response at the alternating current.

The fact that change of impedance while changing frequency depends upon a composition of the substance, allows detecting changes and influence of each component on resultant impedance of the substance at different frequencies.

After determining weight coefficients of influence of relevant components on resultant impedance of the substance on each of operating frequencies of resonance sensors, it is possible based on sensor readings, solving a system of linear equations to obtain information about a concentration of studied components.

A right choice of operating frequencies of sensors makes a great impact on accuracy of this method.

By scanning in a wide range of frequencies it is necessary to determine the most inherent for each component frequency domains, i.e frequencies, at which a component gives the largest response.

Traditional impedance spectroscopy in its researches uses ac voltage source, which affects the studied sample by a contact method, whereas electric current appears in the circuit, a value and phase shift of which depend upon impedance of the sample.

Results are displayed as a rule as Lissajous patterns or Nyquist diagrams.

With such researches it is difficult to achieve high sensitivity and accuracy of measurements. The proposed methodology, in which measurement of impedance is made using resonance circuits, has a significantly higher sensitivity and accuracy, furthermore it is contactless.

There exist certain technical difficulties of creating an oscillating circuit with a resonance frequency readjusted in a wide range, therefore it will be necessary to use traditional Magnetic resonance spectroscopy - impedance spectroscopy for searching for frequencies "inherent" for components.

When inherent frequencies are found and resonance sensors are created for these frequencies, a system of component monitoring created based on these sensors will have exclusive sensitivity and accuracy. 


\section{Interference immunity}

Such "mechanic" parameters as VISCOSITY, DENSITY, TRANSPARENCY, PRESSURE (if a medium is incompressible) shall not make any impact on measured electric parameters of a substance.

MOTION SPEED IN A PIPLELINE and TURBULENCE - these phenomena are too slow in order to make an impact on "megaherz" processes of impedance measurement. HARDNESS is a chemical parameter, which is fully determined by components that are included into the substance.

Temperature, as a rule, makes an impact on a value of impedance, but measurement of temperature and its recording while impedance measuring is not a complicated technical task.

1. Principled foundations of secure coding of optical storage media or information tanks, predominantly in the form of a disk, transparent for luminous flux, coming from an exit optical system of a single-mode laser diode, having standard required dimensions, - outer diameter, - 120 millimeters, and thickness, -1,2 millimeter.

The disk is pasted of two halves, each 0,6 millimeter thick; a coating is applied on one of halves of the disk on a ring, the outer dimeter of which is 120 millimeters, and the inner dimeter of which is 118 millimeters; the thickness of the coating varies in a range from 1 micron to 10 microns with an interval 100 angstrom.

Suh accuracy is fully ensured by properties and parameters of a technology of rapid electrochemical coatings and as well as ensures high accuracy of identification in operation, not introducing mistakes related to inaccuracy of code measurements.

1.1. Conceptual foundations of coding reside in the following principle: - a coding signal is produced from a reaction of a sensing unit or a group of sensing units to a thickness of a ring coating on a disk, comparison of the obtained signal to a statistical reference of this signal, - equivalent of the resonance reaction of sensing units to the thickness of the coating, specific indicators of the coating material, conductivity of the coating material, density of the coating material, electric resistance of the coating material.

1.2. Instead of one of points of the group combination, a signal from a decoding sensing unit of the secure coding system is introduced to the servo-marking system of the formatted disk, which, as a rule, has a type of group combinations of servo-points on information tracks of the disk, and in case of coincidence of an integrated signal from three sensing units with pre-set parameters of the signal, the servo system of a disk drive starts orienting a focus of the laser on the information track, and thus the system starts the reading or recording process on the optical disk.

1.3. In case of non-coincidence of the signal from sensing units with a statistical form of the signal in the memory of the disk drive processor, the servo system of the disk drive does 
not orient and does not stabilize a trajectory of a beam focus of the laser diode on the information track of the disk and reading or recording on the disk become impossible.

\section{OPTIONS OF IDENTIFICATION OF A DISK IN A DISK DRIVE:}

2.1. Identification of a disk in a disk drive can be run using measurement of a coating thickness in a real-time mode, comparison of results of measurement to a statistical value of this parameter stored in the disk drive processor and delivery of the signal to a comparator in the disk drive processor.

2.2. The identification process can be run during disk rotation or during installation of the disk to the disk drive.

2.3. During identification during installation of the disk to the disk drive, negative results of identification do not allow switching of any structure of the disk drive, and on the contrary a positive signal of identification switches on necessary structures of the disk drive.

2.4. Similar logics and procedure of operation take place in the coding and decoding system of one-use tool in different devices and technical systems of any level.

2.5. Such design interrelation substantially standardizes all technological transitions of the identification, coding and decoding process, irrespective of a specific type and scope of application of the product, and only depending on design features and characteristics of a special unit for coding and decoding.

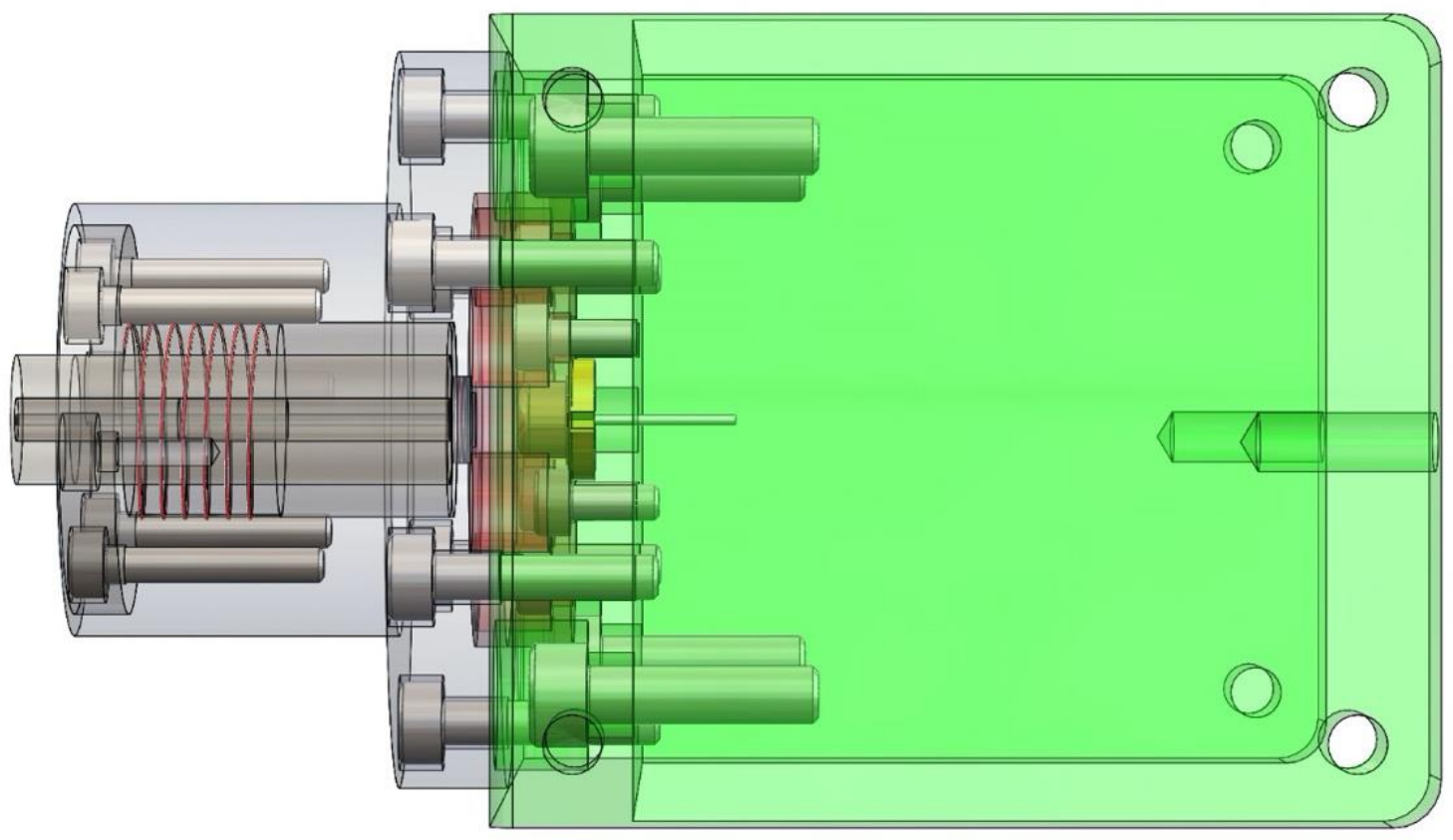

Figure 16, - a three-dimensional model of a docking device of a laser endoscope for installation and identification of one-use tool is also shown in the drawing. The model is shown in a transparent version, top view, where a system of functional interaction is seen between a laser diode and an optical cable, as an axis of one-use tool. The solenoid of the 
sensing unit from the signal of which coding and decoding of one-use tool is performed is visible

\section{DESIGN OPTIONS OF DISK DRIVES:}

3.1. Elements of the secure system of resonance coding - decoding can be without any design or circuit limitations, embedded to any existing today design of the disk drive, implementing all known technologies of optical memory.

3.2. Existing disk drives can also be modified for assembling of the system of microsensors, by insetting a sensor micromodule to a bearing structure of the disk drive casing.

3.3. If necessary, a coating can be performed out on already existing disks.

\section{APPROXIMATE PROCESSING ROUTE OF MANUFACTURING A DISK WITH A CODING COATING:}

4.1. Special technologies and equipment are not required for manufacturing an optical disk with a secure coding coating.

4.2. Upgraded processing equipment, which is currently used, can be used for manufacturing.

4.3. Application of a coding coating can be combined with manufacturing a copy of the disk in a press mould using a master disk with an identification point in the formatted servomarking system, which in such manner will be pressed on each information track, - and there are more than 37000 in an ordinary optical disk.

\section{OPTIONS OF USING DISKS WITH A SECURE COATING IN SYSTEMS OF OPTICAL MEMORY OF CORPORATE CLIENTS:}

5.1. An approximate scheme of using disks with protective coding-decoding in corporate clients provides for manufacturing a certain amount of disks for each such client with parameters of thickness and coordinates of microsensors inherent only to this client.

5.2. Design and technical characteristics of a sensor micromodule can also be modernized based on client's wishes, but in accordance with control parameters of the secure coding coating on disks. 


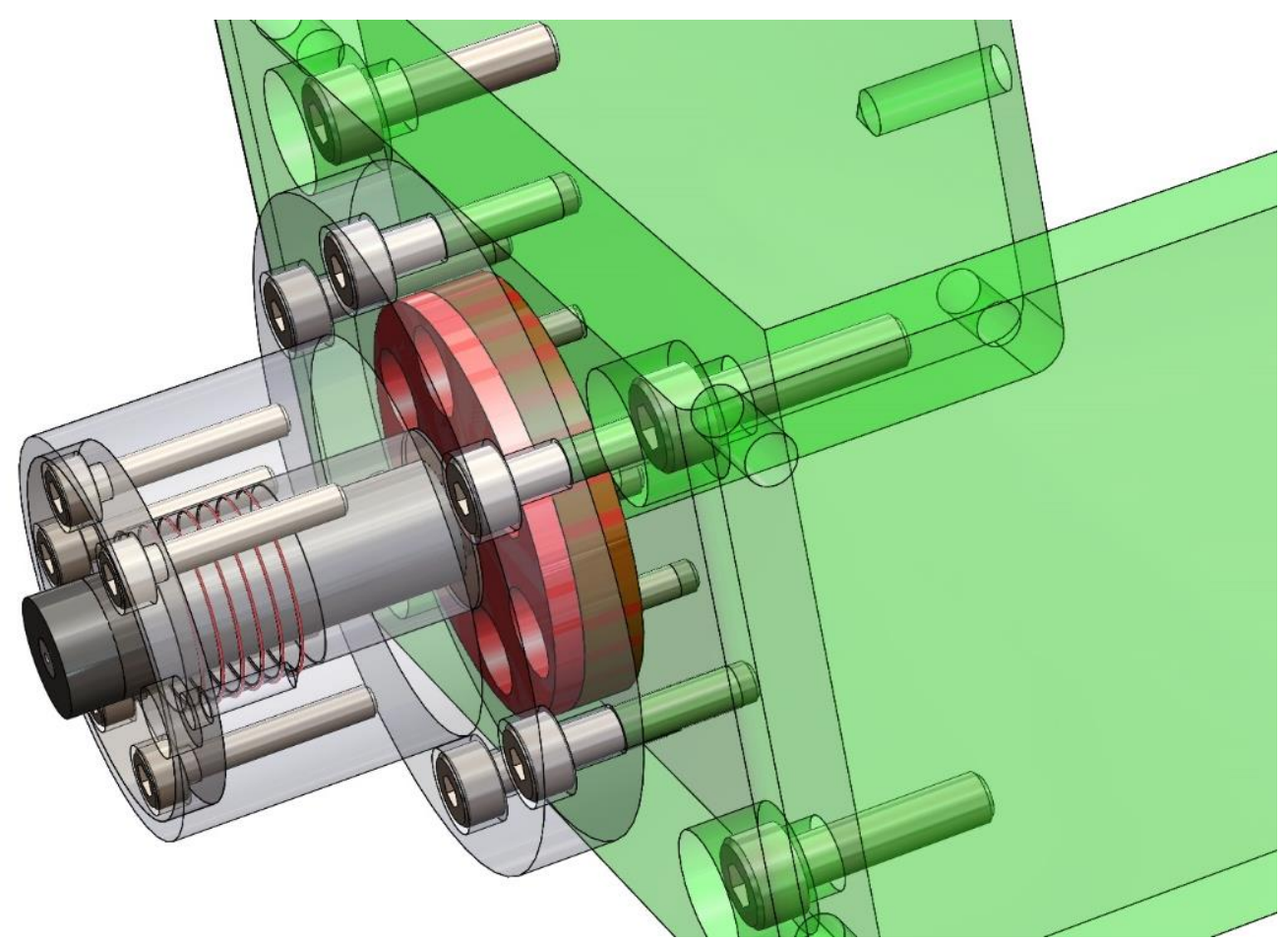

Figure 17, - a three-dimensional model of a docking device of a laser endoscope for installation and identification of one-use tool is also shown in the drawing. The model is shown in a transparent version, isometric view, where a system of functional interaction is seen between a laser diode and an optical cable, as an axis of one-use tool. The solenoid of the sensing unit from the signal of which coding and decoding of one-use tool is performed is visible

\section{OPTIONS OF USING DISKS WITH SECURE CODING IN SYSTEMS OF HOUSEHOLD RADIO TECHNOLOGY:}

6.1. Disks with secure coding can be used in Blu-Ray and HD DVD systems.

Apart from that, the system of secure coding can be applied in new developments and technologies of optical digital memory including disks with especially high density of recording, multi-layer disks, monolithic optical disks with the memory capacity 1 and more terabyte.

6.2. While manufacturing disks, necessary indication to servo-marking can be introduced in the pressing process.

A servodrive of the disk drive starts orientation of a focal point of the laser beam only in case of coincidence of a coding signal from the coding and decoding system produced by the system of three micro-sensors, which using magnetic resonance methods, compare a thickness of the coating to a reference and in case of coincidence of parameters of the signal with the reference at least in two sensors, the obtained signal is added to the system of symbols and marking points of servo-marking, reading which the servodrive 
of the disk drive starts stabilizing a focus of the laser on a necessary track in a field of disk recording.

\section{OPTIONS OF USING DISKS WITH A SECURE COATING IN PERSONAL COMPUTERS:}

7.1. A manufacturing technology of disks for personal computers is similar to the manufacturing technology of such kind of disks for other options of optical memory.

7.2. A methodology of using disks with secure coding is built up based on a type of a computer, extent of its saturation and capacity, speed and etc.

7.3. Especially important is a possibility of using processes and technology of secure coding in created hybrid disks, combining in itself a hard disk with an optical disk.

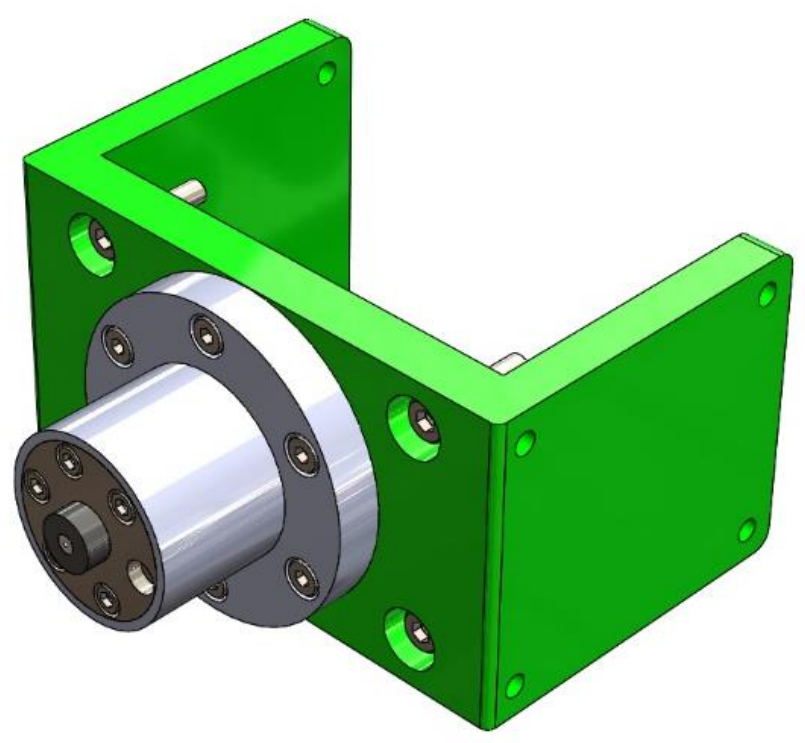

Figure 18, - a three-dimensional model of the casing of the plug-in connector docked to the main detail bearing the laser diode (designated with the green color in the drawing) is shown in the drawing

\section{Proposal on the system of obtaining professional information from Internet}

An optical disk is the main tool, on which a coding coating is applied in a ring zone, in which there is no information recording.

A microsensor is an auxiliary tool, which is embedded into the disk drive. 
A signal from the microsensor is produced while measuring a thickness of the coating; accuracy of measurement, - 100 angstrom and this is value for which each group of disks differ from the other group.

A signal from the microsensor is a code for entering arrays of information placed in Internet.

A software shall make it possible to identify a signal from a microsensor and in case of coincidence of the signal with the reference one to open arrays of information and in the process of its downloading to continue controlling fidelity of the signal till completion of the information downloading process.

This enables preventing replacement of a disk during recording to a non-licensed one.

It is impossible to falsify such disk, since the thickness of the coating is determined during manufacturing and, even having such disk, it is impossible to use it, without a microsensor configured to a strictly definite nature of the signal.

Disks and sensors can be manufactured at any existing today production facility of optical disks; disks can be manufactured in series by 100 - 250 pieces with a similar thickness of the coding tape and with a set of sensors.

Every user may acquire one or several series of disks and use them while working with Internet.

In the same principle programs and other information can be sent to users, only in the inverse order, which warrants full confidentiality and protection while being in Internet from unauthorized messages and viruses.

This is very general information if course, if you readers consider it deserving attention, then the author could elaborate this project.

Taking into account the fact that the mechanical part of this project is as a matter of principle implemented, this project is a software, which may become a basis of the project in this direction. 


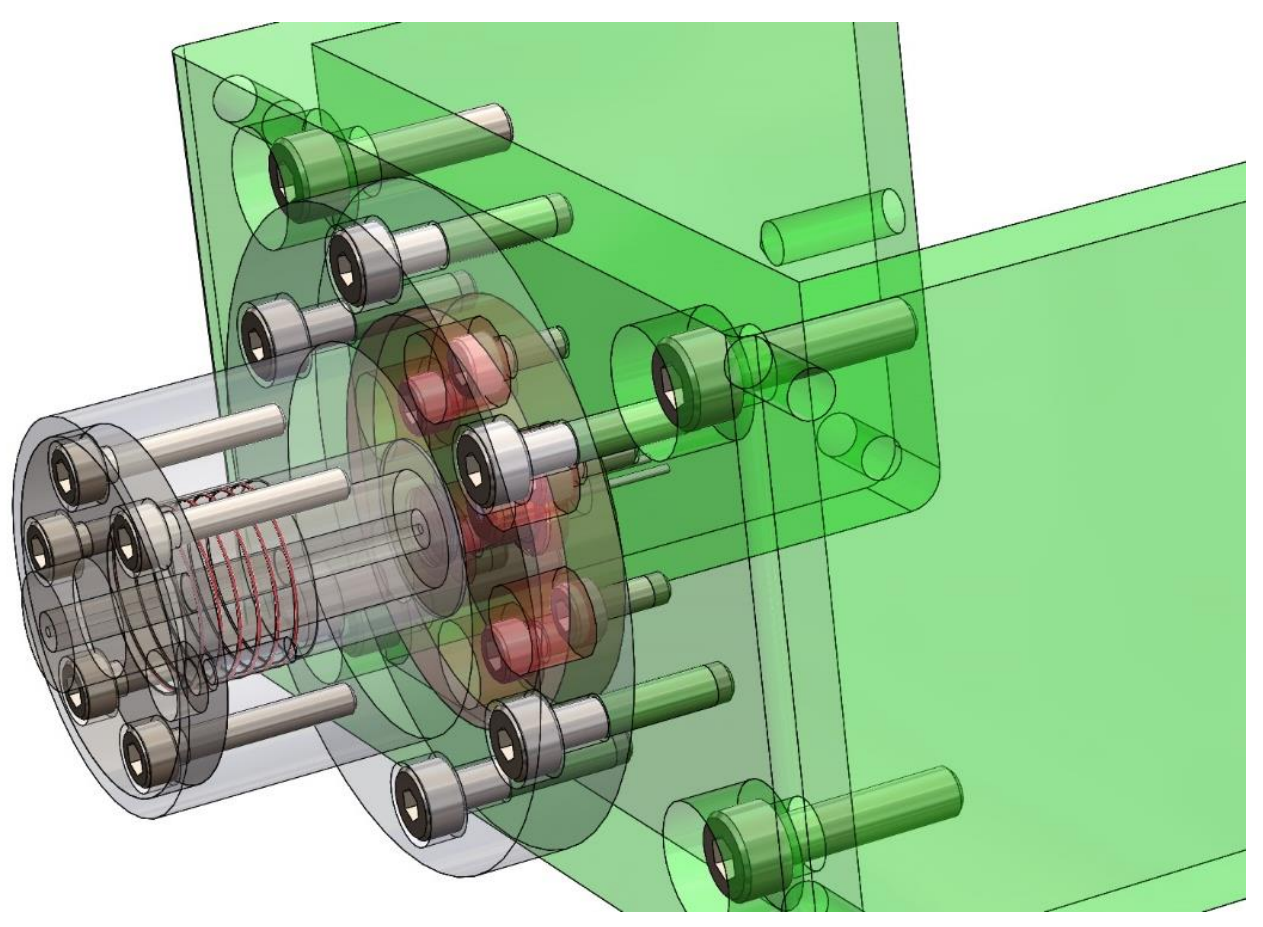

Figure 19, - a coaxial casing of a plug-in connector with a coding and decoding device as a coaxially installed solenoid is shown in the drawing, which jointly with an impulse generator produces a coding and decoding signal and after its passage sorts and transmits a resonance signal to an analytical unit

\section{Advantages of the proposed technology, responding to the essence of problems detected on the market of systems of optical storage media:}

1. There is a variety of options of thicknesses of coding coatings, which allow having a variety of options of a security code, as distinguished from well-known technologies, which have only one option of the code.

2. In the process of application of a coating, a control technology is used fully identical to the decoding technology, which allows fully controlling quality of coding in the process of disk manufacturing, without removal of the disk from a conveyor, as distinguished from existing technologies, in which a disk for control should be removed from the conveyor and installed in a control device.

Thus, control is selective, and in the proposed technology, - 100\% control, which excludes production of defective disks, which are found in existing technologies only during operation.

3. In the proposed technology there is a possibility of coding all categories and types of disks irrespective of a format of recording and reading, as distinguished from existing technologies, in which coding depends upon a format of recording and reading of the disk. 
4. In the proposed technology a coding coating can be a basis for a personal secret code or cipher, which existing technologies do not have.

5. In the proposed technology a sensing unit of decoding and identification is mobile and may have several options of delivery, including an autonomous option, not related to a disk drive, and in the existing technologies the decoding system is installed only in disk drives; thus it is possible to control availability and correctness of coding only in the process of installation of the disk to the disk drive, and in the proposed technology it is possible to control and identify a code out of the disk drive, for example in shops or in entrance control posts of enterprises and institutions, which is especially important for ensuring full nondisclosure mode.

6. In the proposed technology decoding excludes any dependence upon optical systems of the disk drive, but results of decoding may change operation of optical systems, for example a servo drive for orientation and control of a focus position of a reading or recording laser, as distinguished from existing technologies, in which a decoding process fully depends upon optical elements of the disk drive, which complicates its design and severely reduces reliability.

7. The proposed technology has several hierarchies of a fundamental operating scheme, has a flexible algorithm and can be built into any security system of optical memory including to hybrid information-carrying media, which have apart from an optical component, media constructed on other basic principles; existing technologies do no have specified flexibility.

8. The proposed technology allows using a code of a disk as an entrance password for entering professional arrays of information of Internet, which existing technologies do not have.

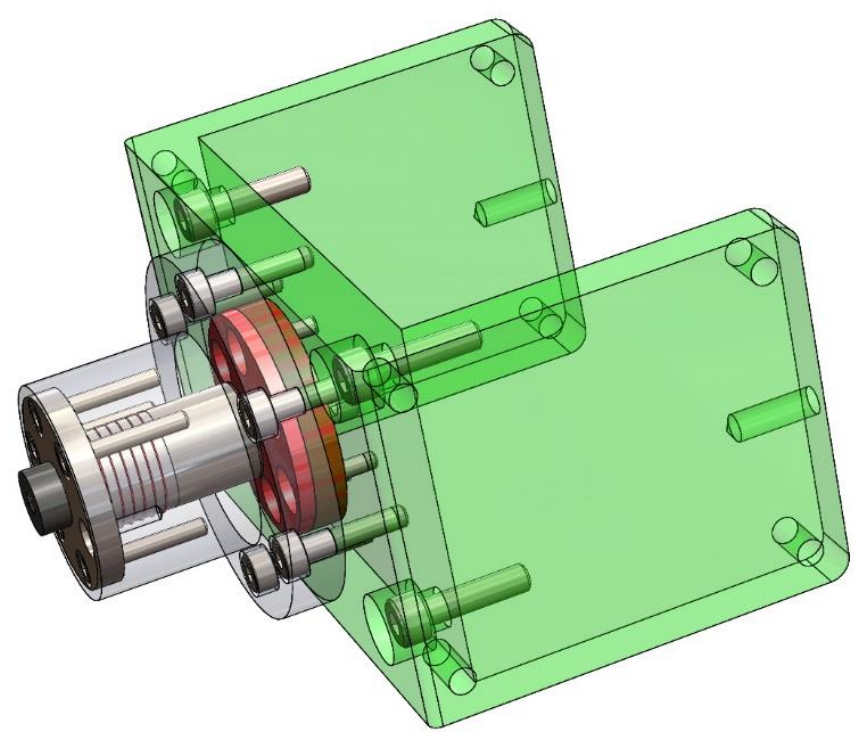

Figure 20, - a module of a laser diode with a coding and decoding plug, containing a sensing unit-solenoid located coaxially to a plug connector of one-use tool is shown in the 
drawing, notably that the connector plug is made as a plug, a wall thickness of which is a key to a ciphering code, which is identified using resonance spectroscopy

Technology and design solution of such an integrative sensing unit, allows its building into practically any technical system, where connection, for example of one-use tool is performed using a plug-in connector.

The following general marketing strategy is developed preliminarily according to results of a complex analysis using algorithms of the patenting and licensing strategy.

\section{Preliminary determination of a core product of the project ad its functional and operating features and characteristics:}

1. The main sector of the market, - corporate clients:

- Banks and financial companies;

- Industrial corporations;

- Scientific Research Laboratories;

- Transport companies, railway station buildings, airports and sea ports;

○ Large distribution networks;

- Municipal services;

- Governmental organizations and institutions;

- Large medical institutions;

- Insurance companies;

- Branches of arms of armed forces;

$\circ$ Police and intelligence services.

2. Product characteristic (as an example):

- A product of the project (main), - an optical disk with a coding ring with an embedded sensor module, as a rule consisting of three microsensors;

- If necessary, the sensor module can be supplied without a disk drive;

- If necessary, a company running the project, can provide services for corporate clients, organizing bringing a coding system and information security into action, - on a turnkey basis. 


\title{
LIST OF REFERENCES, PATENT AND LICENSE MATERIALS
}

\section{ANNEX 1}

United States Patent Application

20190281321

Kind Code

ZHAO; Xin ; et al.

September 12, 2019

\section{METHOD AND APPARATUS FOR VIDEO CODING}

\begin{abstract}
Aspects of the disclosure provide method and apparatus for video coding. In some examples, an apparatus includes processing circuitry. The processing circuitry determines an intra prediction mode for generating a prediction image of a current block, determines one or more primary transforms according to signaling information that is extracted from a coded video bitstream, and determines a secondary transform according to the determined intra prediction mode and the determined one or more primary transforms. The processing circuitry also reconstructs a residue image of the current block based on one or more coefficients of an input block extracted from the coded video bitstream, the determined one or more primary transforms, and the determined secondary transform. The processing circuitry then reconstructs an image of the current block based on the prediction image and the residue image of the current block.
\end{abstract}

\section{ANNEX 2}




\title{
SHARING OF MOTION VECTOR IN 3D VIDEO CODING
}

\begin{abstract}
Joint coding of depth map video and texture video is provided, where a motion vector for a texture video is predicted from a respective motion vector of a depth map video or vice versa. For scalable video coding, depth map video is coded as a base layer and texture video is coded as an enhancement layer(s). Inter-layer motion prediction predicts motion in texture video from motion in depth map video. With more than one view in a bitstream (for multiview coding), depth map videos are considered monochromatic camera views and are predicted from each other. If joint multiview video model coding tools are allowed, inter-view motion skip is used to predict motion vectors of texture images from depth map images. Furthermore, scalable multiview coding is utilized, where inter-view prediction is applied between views in the same dependency layer, and inter-layer (motion) prediction is applied between layers in the same view.
\end{abstract}

\section{ANNEX 3}

United States Patent Application

20190273948

Kind Code

Yin; Hujun ; et al.

September 5, 2019

METHOD AND SYSTEM OF NEURAL NETWORK LOOP FILTERING FOR VIDEO CODING

Abstract

A method, system, medium, and article provide neural network loop filtering for video coding with multiple alternative neural networks.

\section{ANNEX 4}

United States Patent Application

20190273932

Kind Code

HAQUE; MUNSI ; et al.

September 5, 2019

VIDEO CODING SYSTEM WITH TEMPORAL SCALABILITY AND METHOD OF OPERATION THEREOF 


\begin{abstract}
A method of operation of a video coding system includes: receiving a video bitstream; extracting a video syntax from the video bitstream; extracting a hypothetical reference decoder (HRD) fixed syntax from the video syntax; extracting a HRD variable syntax from the video syntax; extracting a temporal layer from the video bitstream based on the HRD fixed syntax and the HRD variable syntax; and forming a video stream based on the temporal layer for displaying on a device.
\end{abstract}

\title{
ANNEX 5
}

United States Patent Application

20190268098

Kind Code

A1

CHUN; Jinyoung ; et al.

August 29, 2019

DATA TRANSMISSION METHOD IN WIRELESS COMMUNICATION SYSTEM AND

DEVICE THEREFOR

\begin{abstract}
A downlink (DL) multi-user (MU) transmission method in a wireless local area network (WLAN) system, the DL MU transmission method including receiving a DL MU physical protocol data unit (PPDU) including a physical preamble and a data field from STA (Station) and transmitting the ACK frames in response to the DL MU PPDU to STA. In addition, the data field includes at least one medium access control (MAC) protocol data unit (MPDU), the at least one MPDU includes a MAC header and a MAC frame body, wherein the MAC header includes acknowledge (ACK) indication information, the ACK indication information includes frequency resource allocation information for an uplink (UL) MU orthogonal frequency division multiple access (OFDMA) transmission of ACK frames and modulation and coding scheme (MCS) level information, and the frequency resource allocation information includes an index value indicating a resource unit allocated for the UL MU OFDMA transmission of the ACK frames, and the resource unit corresponds to a 26-tone resource unit, a 52-tone resource unit, a 106-tone resource unit, a 242-tone resource unit, a 484-tone resource unit, or a 996-tone resource unit.
\end{abstract}

\section{ANNEX 6}

United States Patent Application

20190268016

Kind Code

JANSSON TOFTG RD; Tomas ; et al.

August 29, 2019 


\begin{abstract}
A method for partitioning of input vectors for coding is presented. The method comprises obtaining of an input vector. The input vector is segmented, in a non-recursive manner, into an integer number, N.sup.SEG, of input vector segments. A representation of a respective relative energy difference between parts of the input vector on each side of each boundary between the input vector segments is determined, in a recursive manner. The input vector segments and the representations of the relative energy differences are provided for individual coding. Partitioning units and computer programs for partitioning of input vectors for coding, as well as positional encoders, are presented.
\end{abstract}

\title{
ANNEX 7
}

United States Patent Application

20190261010

Kind Code

A1

Luo; Ning ; et al.

August 22, 2019

METHOD AND SYSTEM OF VIDEO CODING WITH REDUCED SUPPORTING DATA SIDEBAND BUFFER USAGE

\begin{abstract}
Methods, systems, and articles of video coding with reduced supporting data sideband buffer usage.
\end{abstract}

\section{ANNEX 8}

United States Patent Application

20190253706

Kind Code

ZHAO; Liang ; et al.

August 15, 2019

METHOD AND APPARATUS FOR USING AN INTRA PREDICTION CODING TOOL FOR INTRA PREDICTION OF NON-SQUARE BLOCKS IN VIDEO COMPRESSION

\begin{abstract}
A method for video decoding includes determining whether an angular intra prediction mode for a current block is a wide angle mode that is spaced apart from a vertical mode and a horizontal mode by an angular distance that is more than a predetermined threshold. The method further includes, in response to the determination that the angular intra prediction mode
\end{abstract}


is the wide angle mode, determining a non-wide angle mode that corresponds to the wide angle mode, the non-wide angle mode being spaced apart from the vertical mode or the horizontal mode by an angular distance that is less than or equal to the predetermined threshold. The method includes determining whether a coding tool is specified for the determined corresponding non-wide angle mode, and, in response to the determination that the coding tool is specified for the determined corresponding non-wide angle mode, using the coding tool in a decoding process.

\title{
ANNEX 9
}

United States Patent Application

20190181979

Kind Code

Wang; Ying ; et al.

June 13, 2019

\section{ADJUSTED FRACTALLY ENHANCED KERNEL POLAR CODES FOR ACHIEVABLE SIGNAL-TO-NOISE RATIO SPIKE MITIGATION}

\begin{abstract}
Methods, systems, and devices for wireless communications are described. In some systems, wireless devices may implement adjusted fractally enhanced kernel polar coding. An encoder may receive a number of information bits and a block size for transmission, and may append an additional number of information bits to the information bits for transmission. The encoder may perform a recursive bit allocation process to allocate the aggregate set of information bits between a set of sub-blocks based on mutual information metrics. To obtain the correct number of information bits and block size, the encoder may remove a number of information bits equal to the number of appended additional bits (e.g., from a first half of the sub-blocks), assign the remaining information bits to bit channels in each sub-block, and block puncture a set of bits (e.g., from the first half). The resulting codeword may mitigate occurrences of achievable signal-to-noise ratio (SNR) spikes.
\end{abstract}

\section{ANNEX 10}

United States Patent Application

20190182503

Kind Code

Tsai; Yi-Ting ; et al. 
A method and an image processing apparatus for video coding are proposed. The method is applicable to an image processing apparatus and includes the following steps. A current coding unit is received, and the number of control points of a current coding unit is set, where the number of control points is greater than or equal to 3. At least one affine model is generated based on the number of control points, and an affine motion vector corresponding to each of the at least one affine model is computed. A motion vector predictor of the current coding unit is computed based on the at least one motion vector so as to accordingly perform interprediction coding on the current coding unit.

\title{
ANNEX 11
}

United States Patent Application

20190190578

Kind Code

A1

Mittal; Udar ; et al.

June 20, 2019

\section{CODING MAIN BEAM INFORMATION IN CSI CODEBOOK}

\begin{abstract}
Apparatuses, methods, and systems are disclosed for preparing a channel state information ("CSI") codeword. One apparatus includes a processor and a transceiver configured to communicate 805 with a transmit-receive point ("TRP") over a radio access network using spatial multiplexing, wherein multiple transmission layers are transmitted at a time, each transmission layer comprising multiple beams. The processor identifies a main beam for each of the multiple transmission layers and determines whether the main beams of each transmission layer are the same. The processor prepares a CSI codeword, wherein the CSI codeword comprises a first bit indicating whether the main beams of each transmission layer are the same, a first set of bits coding the main beams, and a second set of bits coding the remaining beams. The transceiver transmits the CSI codeword to the TRP.
\end{abstract}

\section{ANNEX 12}

United States Patent Application

20190200025

Kind Code

A1

Li; Bin ; et al.

June 27, 2019

\section{CODED-BLOCK-FLAG CODING AND DERIVATION}

\begin{abstract}
Techniques for coding and deriving (e.g., determining) one or more coded-block-flags associated with video content are described herein. A coded-block-flag of a last node may be
\end{abstract}


determined when coded-block-flags of preceding nodes are determined to be a particular value and when a predetermined condition is satisfied. In some instances, the predetermined condition may be satisfied when log.sub.2(size of current transform unit) is less than log.sub.2 (size of maximum transform unit) or log.sub.2(size of current coding unit) is less than or equal to log.sub.2(size of maximum transform unit) +1 . The preceding nodes may be nodes that precede the last node on a particular level in a residual tree.

\title{
ANNEX 13
}

United States Patent Application

20190222863

Kind Code

HANNUKSELA; Miska Matias ; et al.

July 18, 2019

\author{
VIDEO CODING AND DECODING
}

\begin{abstract}
There is disclosed a method, an apparatus, a server, a client and a non-transitory computer readable medium comprising a computer program stored therein for multi view video coding and decoding. View random access (VRA) pictures or access units are coded into a multiview bitstream. VRA pictures enable starting the decoding of a subset of the views present in the bitstream. The views selected to be accessible in VRA pictures are alternated in successive VRA pictures so that all views are gradually reconstructed when two or more VRA pictures have been decoded.
\end{abstract}

\section{ANNEX 14}

United States Patent Application

20190200026

Kind Code

LAINEMA; Jani ; et al.

June 27, 2019

\section{APPARATUS, A METHOD AND A COMPUTER PROGRAM FOR VIDEO CODING}

\begin{abstract}
There is disclosed an apparatus, a method and a computer program for video coding. The apparatus comprises a selector configured for selecting a pixel for prediction; a projection definer configured for determining a projection of said pixel to a set of reference pixels; and a prediction definer configured for selecting one or more reference pixels from said set of reference pixels on the basis of said projection, and using said selected one or more reference pixels to obtain a prediction value for said pixel to be predicted.
\end{abstract}




\title{
ANNEX 15
}

United States Patent Application

20190208222

Kind Code

UGUR; Kemal ; et al.

July 4, 2019

\section{APPARATUS, A METHOD AND A COMPUTER PROGRAM FOR VIDEO CODING AND DECODING}

\begin{abstract}
There are disclosed various methods, apparatuses and computer program products for video encoding and decoding. In other embodiments, there is provided a method, an apparatus, a computer readable storage medium stored with code thereon for use by an apparatus, and a video encoder, for encoding a scalable bitstream, to provide indicating an encoding configuration, where only samples and syntax from intra coded pictures of base layer is used for coding the enhancement layer pictures. In other embodiments, there is provided an apparatus, a computer readable storage medium stored with code thereon for use by an apparatus, and a video decoder, for decoding a scalable bitstream, to receive indications of an encoding configuration, where only samples and syntax from intra coded pictures of base layer is used for coding the enhancement
\end{abstract}

\section{ANNEX 16}

United States Patent Application

20190222859

Kind Code

CHUANG; Tzu-Der ; et al.

July 18, 2019

\section{METHOD AND APPARATUS OF CURRENT PICTURE REFERENCING FOR VIDEO CODING}

\begin{abstract}
A method and apparatus for a video coding system with the current picture referencing (CPR) mode enabled are disclosed. According to one method, the luma and chroma blocks of the current image are jointly coded using a same coding unit (CU) structure if the CPR mode is selected for the luma and the chroma blocks. Alternatively, if the luma and chroma components are partitioned into the luma and the chroma blocks separately using separate $\mathrm{CU}$ structures, the luma and chroma blocks are encoded or decoded using a coding mode selected from a coding mode group excluding the CPR mode. According to another method, the luma and chroma blocks of the current image are coded separately using a different CU structure if the
\end{abstract}


CPR mode is selected for the luma and chroma blocks. In yet another method, reconstructed reference data is disclosed for the CPR mode with CU equal to PU.

\title{
ANNEX 17
}

United States Patent Application

20190230365

Kind Code

Tanner; Jason ; et al.

July 25, 2019

\section{VIDEO CLUSTER ENCODING FOR MULTIPLE RESOLUTIONS AND BITRATES WITH PERFORMANCE AND QUALITY ENHANCEMENTS}

\begin{abstract}
Techniques related to video cluster encoding are discussed. Such techniques include encoding the video at a first resolution and first bitrate, translating block based coding parameters corresponding thereto to block based encode controls for encode of the same video at a second resolution or a second bitrate, and encoding the video at the second resolution and/or bitrate using the encode controls.
\end{abstract}

\section{ANNEX 18}

United States Patent Application

20190246126

Kind Code

Abbas; Adeel ; et al.

August 8, 2019

APPARATUS AND METHODS FOR VIDEO COMPRESSION USING MULTIRESOLUTION SCALABLE CODING

\begin{abstract}
Apparatus and methods for digital video data compression via a scalable, multi-resolution approach. In one embodiment, the video content may be encoded using a multi-resolution and/or multi-quality scalable coding approach that reduces computational and/or energy load on a client device. In one implementation, a low fidelity image is obtained based on a first full resolution image. The low fidelity image may be encoded to obtain a low fidelity bitstream. A second full resolution image may be obtained based on the low fidelity bitstream. A portion of a difference image obtained based on the second full resolution image and the first full resolution may be encoded to obtain a high fidelity bitstream. The low fidelity bitstream and the high fidelity bitstream may be provided to e.g., a receiving device.
\end{abstract}


ANNEX 19

United States Patent Application

20190246118

Kind Code

A1

YE; Jing ; et al.

August 8, 2019

METHOD AND APPARATUS FOR VIDEO CODING IN MERGE MODE

\begin{abstract}
A method for video coding using a merge mode by a decoder or encoder. An embodiment of the method includes receiving a current block having a block size, setting a grid pattern based on the block size of the current block, wherein the grid pattern partitions a search region adjacent to the current block into search blocks, and a size of the search blocks is determined according to the block size of the current block, and searching for one or more spatial merge candidates from candidate positions in the search blocks to construct a candidate list that includes the one or more spatial merge candidates.
\end{abstract}

\title{
ANNEX 20
}

United States Patent Application

20190034583

Kind Code

Kartalov; Emil P. ; et al.

January 31, 2019

SIGNAL ENCODING AND DECODING IN MULTIPLEXED BIOCHEMICAL ASSAYS

\begin{abstract}
This disclosure provides methods, systems, compositions, and kits for the multiplexed detection of a plurality of analytes in a sample. In some examples, this disclosure provides methods, systems, compositions, and kits wherein multiple analytes may be detected in a single sample volume by acquiring a cumulative measurement or measurements of at least one quantifiable component of a signal. In some cases, additional components of a signal, or additional signals (or components thereof) are also quantified. Each signal or component of a signal may be used to construct a coding scheme which can then be used to determine the presence or absence of any analyte.
\end{abstract}

\section{ANNEX 21}

United States Patent Application

20170236521

Kind Code 


\title{
Chebiyyam; Venkata Subrahmanyam Chandra Sekhar ; et al.
}

\section{ENCODING OF MULTIPLE AUDIO SIGNALS}

\begin{abstract}
A device includes an encoder and a transmitter. The encoder is configured to determine a mismatch value indicative of an amount of temporal mismatch between a reference channel and a target channel. The encoder is also configured to determine whether to perform a first temporal-shift operation on the target channel at least based on the mismatch value and a coding mode to generate an adjusted target channel. The encoder is further configured to perform a first transform operation on the reference channel to generate a frequency-domain reference channel and perform a second transform operation on the adjusted target channel to generate a frequency-domain adjusted target channel. The encoder is also configured to estimate one or more stereo cues based on the frequency-domain reference channel and the frequency-domain adjusted target channel. The transmitter is configured to transmit the one or more stereo cues to a receiver.
\end{abstract}

